\title{
Pitch angle scattering of an energetic magnetized particle by a circularly polarized electromagnetic wave
}

P. M. Bellan

Citation: Phys. Plasmas 20, 042117 (2013); doi: 10.1063/1.4801055

View online: http://dx.doi.org/10.1063/1.4801055

View Table of Contents: http://pop.aip.org/resource/1/PHPAEN/v20/i4

Published by the American Institute of Physics.

\section{Additional information on Phys. Plasmas}

Journal Homepage: http://pop.aip.org/

Journal Information: http://pop.aip.org/about/about_the_journal

Top downloads: http://pop.aip.org/features/most_downloaded

Information for Authors: http://pop.aip.org/authors

\section{ADVERTISEMENT}

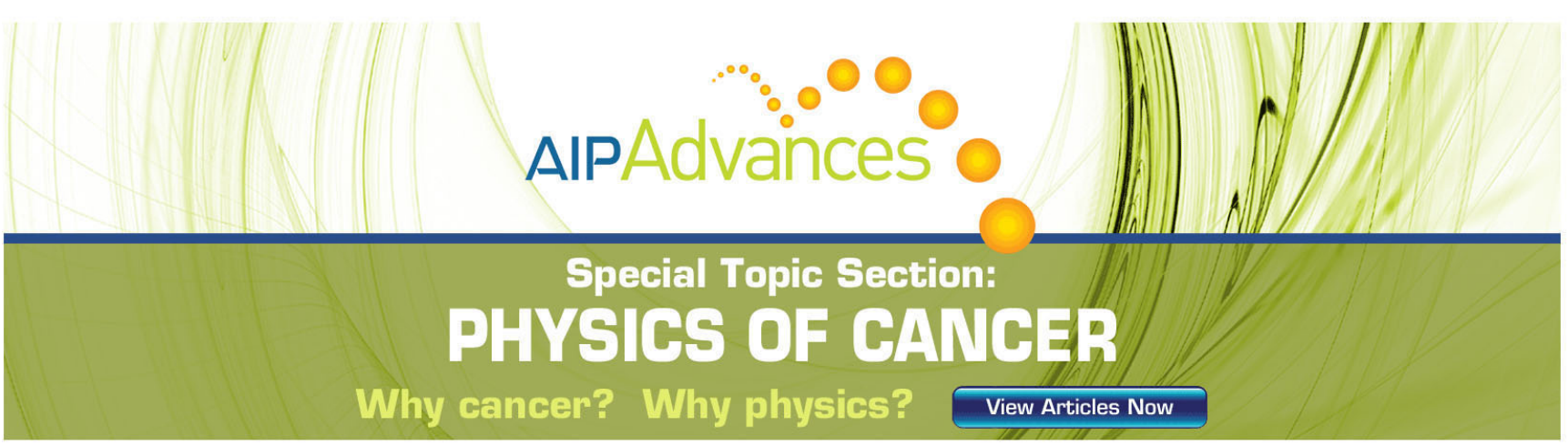




\title{
Pitch angle scattering of an energetic magnetized particle by a circularly polarized electromagnetic wave
}

\author{
P. M. Bellan \\ Applied Physics, Caltech, Pasadena, California 91125, USA
}

(Received 7 February 2013; accepted 26 March 2013; published online 19 April 2013)

\begin{abstract}
The interaction between a circularly polarized wave and an energetic gyrating particle is described using a relativistic pseudo-potential that is a function of the frequency mismatch. Analysis of the pseudo-potential provides a means for interpreting numerical results. The pseudo-potential profile depends on the initial mismatch, the normalized wave amplitude, and the initial angle between the wave magnetic field and the particle perpendicular velocity. For zero initial mismatch, the pseudopotential consists of only one valley, but for finite mismatch, there can be two valleys separated by a hill. A large pitch angle scattering of the energetic electron can occur in the two-valley situation but fast scattering can also occur in a single valley. Examples relevant to magnetospheric whistler waves show that the energetic electron pitch angle can be deflected $5^{\circ}$ towards the loss cone when transiting a $10 \mathrm{~ms}$ long coherent wave packet having realistic parameters. (C) 2013 AIP Publishing LLC [http://dx.doi.org/10.1063/1.4801055]
\end{abstract}

\section{INTRODUCTION}

The interaction between a circularly polarized electromagnetic wave and a charged particle is important in many contexts, with important examples being magnetospheric whistler waves (chorus), ${ }^{1-11}$ Alfvén waves, ${ }^{12,13}$ helicon devices, ${ }^{14,15}$ electron cyclotron masers, ${ }^{16}$ whistler-mediated fast magnetic reconnection, ${ }^{17-19}$ relativistic electron-positron plasmas, ${ }^{20,21}$ and particle accelerators. ${ }^{22-24}$ The interaction can change both the particle energy and pitch angle with the latter change leading to particle escape if the particle is in a magnetic mirror configuration such as Earth's dipole magnetic field. Resonant interaction occurs when

$$
\omega-k v_{z}=\frac{\Omega}{\gamma},
$$

where $\omega$ is the wave frequency, $k$ is the wavenumber along the equilibrium magnetic field, $\Omega=q B / m$ is the cyclotron frequency, $v_{z}$ is the particle velocity component along the equilibrium magnetic field, and $\gamma=\left(1-v^{2} / c^{2}\right)^{-1 / 2}$. It has been proposed by Kennel and Petschek ${ }^{25}$ that this interaction results in a velocity space diffusion wherein energetic electrons are pitch-angle scattered into the loss cone of the Earth's dipole magnetic field and so impact the ionosphere to produce aurora. Kennel and Petschek's model presumes that the wave is incoherent, so that each random step of the diffusive process is set by the time duration for which the resonant condition given by Eq. (1) is satisfied. However, Tsurutani et al. ${ }^{9}$ recently reported spacecraft observations indicating that chorus bursts are actually extremely coherent; these observations raise the question of whether pitch angle scattering can occur in a coherent wave. Furthermore, it is seen that pitch angle scattering will change $v_{z}$ and so destroy the resonance prescribed by Eq. (1).

We explore the detailed wave-particle interaction using an exact fully relativistic analysis. Main results are the demonstration that an energetic particle in a weak coherent wave can experience a large pitch angle deflection and so be scattered into the loss cone and furthermore that large pitch angle transport can occur when Eq. (1) is not initially satisfied.

The wave-particle interaction discussed here differs substantively from the classic interaction between an electrostatic wave and a particle. In the electromagnetic situation, there is no electric field in the wave frame whereas for an electrostatic wave, a static electric field exists in the wave frame and some particles are trapped in the valleys while other particles pass over the hills. ${ }^{26}$ Because there is no electric field in the wave frame, the wave-frame particle kinetic energy is a constant but there is a periodic sloshing back and forth between parallel and perpendicular wave-frame kinetic energies.

We note that other approaches have been previously used to investigate this problem. For example, Bourdier and Gond $^{27}$ used Hamiltonian methods while Qian ${ }^{28,29}$ have sought constants of the motion.

\section{ANALYSIS}

\section{A. Field and particle equations}

We assume that the wave vector is parallel to a uniform background magnetic field in the $z$ direction and assume that $\omega / k<c$ as is true for whistler and Alfvén modes. The wave refractive index is defined as $n=c k / \omega$ so $n>1$.

The ratio of the strength of the wave magnetic field to the background field is defined to be $\kappa$, so the total magnetic field can be written as $\mathbf{B}=B_{0} \hat{z}+\tilde{\mathbf{B}}$ where the wave magnetic field is

$$
\tilde{\mathbf{B}}=\kappa B_{0}[-\hat{x} \sin (k z-\omega t)+\hat{y} \cos (k z-\omega t)] .
$$

Faraday's law requires the associated wave electric field to be

$$
\tilde{\mathbf{E}}=-\frac{\omega}{k} \hat{z} \times \tilde{\mathbf{B}}=\frac{\omega}{k} \tilde{B}(\hat{x} \cos (k z-\omega t)+\hat{y} \sin (k z-\omega t)) .
$$


The charged particle motion is determined by the relativistic Lorentz equation

$$
\frac{d}{d t}(\gamma \beta)=\frac{q}{m}\left(\frac{1}{c} \tilde{\mathbf{E}}+\beta \times \mathbf{B}\right),
$$

where $\beta=\mathbf{v} / c$ and $\gamma=\left(1-\beta^{2}\right)^{-1 / 2}$ and $\mathbf{B}=B_{0} \hat{z}+\tilde{\mathbf{B}}$.

\section{B. Transformation to the wave frame}

The motion will be examined by transforming to a frame, denoted the prime frame, that moves at constant velocity $\mathbf{V}$ with respect to the lab frame. The fields in the prime frame are

$$
\begin{gathered}
\mathbf{E}_{\|}^{\prime}=\mathbf{E}_{\|}, \\
\mathbf{B}_{\|}^{\prime}=\mathbf{B}_{\|}, \\
\mathbf{E}_{\perp}^{\prime}=\gamma_{T}(\mathbf{E}+\mathbf{V} \times \mathbf{B})_{\perp}, \\
\mathbf{B}_{\perp}^{\prime}=\gamma_{T}\left(\mathbf{B}-\frac{1}{c^{2}} \mathbf{V} \times \mathbf{E}\right)_{\perp},
\end{gathered}
$$

where the "transformation gamma" is $\gamma_{T}=1 / \sqrt{1-V^{2} / c^{2}}$. We choose $\mathbf{V}=\hat{z} \omega / k$ in order for the prime frame to be the wave frame, so

$$
\gamma_{T}=1 / \sqrt{1-1 / n^{2}}
$$

Because $n>1, \gamma_{T}$ is real and the transformation is to a physically realizable frame.

We define $\Omega=q B / m$ as the cyclotron frequency of a nonrelativistic particle in the lab frame and $\alpha=\omega / \Omega$ as the normalized wave frequency. Since $\{\mathbf{x}, i c t\}$ and $\{\mathbf{k}, i \omega / c\}$ are four-vectors, it is seen that

$$
k z-\omega t=k \gamma_{T}\left(z^{\prime}+V t^{\prime}\right)-\omega \gamma_{T}\left(t^{\prime}+\frac{V z^{\prime}}{c^{2}}\right)=k^{\prime} z^{\prime},
$$

where $k^{\prime}=k / \gamma_{T}$ is the wavenumber in the wave frame.

Using Eq. (7), it is seen that $\mathbf{E}_{\perp}^{\prime}$ vanishes so there is no electric field in the wave frame. The equation of motion in the wave frame therefore reduces to

$$
\frac{d}{d t^{\prime}}\left(\gamma^{\prime} \beta^{\prime}\right)=\frac{q}{m} \beta^{\prime} \times \mathbf{B}^{\prime},
$$

where $\gamma^{\prime}=1 / \sqrt{1-\beta^{\prime 2}}$. Thus, $\gamma^{\prime} m c^{2}$ is the relativistic energy of the particle in the wave frame. Note that $\gamma, \gamma_{T}$, and $\gamma^{\prime}$ differ and should not be confused with each other.

\section{Relativistic resonant diffusion curve}

Dotting Eq. (11) with $\gamma^{\prime} \beta^{\prime}$ shows that $\gamma^{\prime 2} \beta^{\prime 2} / c^{2}=$ const., which implies $\beta^{\prime 2}=$ const. Hence $\gamma^{\prime}$ is constant, so the waveframe particle relativistic energy is constant. Since $\{\gamma \beta, i \gamma\}$ is a four-vector

$$
\begin{gathered}
\gamma^{\prime} \beta_{\perp}^{\prime}=\gamma \beta_{\perp}, \\
\gamma^{\prime} \beta_{z}^{\prime}=\gamma_{T}\left(\gamma \beta_{z}-\frac{\gamma}{n}\right),
\end{gathered}
$$

$$
\gamma^{\prime}=\gamma_{T}\left(\gamma-\frac{\gamma \beta_{z}}{n}\right)
$$

Because both $\gamma^{\prime}$ and $\gamma_{T}$ are constant, Eq. (14) can be expressed as

$$
\varepsilon=\frac{1-\beta_{z} / n}{\sqrt{1-\beta_{\perp}^{2}-\beta_{z}^{2}}}
$$

where $\varepsilon=\gamma^{\prime} / \gamma_{T}$ is a constant determined by initial conditions. Equation (15) corresponds to Eq. (2.4) of Roberts and Buchsbaum $^{22}$ and is a relativistic extension of the "resonant diffusion curve" used in magnetospheric studies. Because $n>1$ and $\left|\beta_{z}\right|<1$, it is seen that $\varepsilon$ is positive definite. For slow waves and non-relativistic energies, $\varepsilon \rightarrow 1$.

On defining $\Gamma=n^{-2}+\varepsilon^{2}$, Eq. (15) can be written as

$$
\frac{\varepsilon^{2}}{\Gamma} \beta_{\perp}^{2}+\left(\beta_{z}-\frac{1}{n \Gamma}\right)^{2}=\frac{1}{\Gamma}\left(\varepsilon^{2}-1+\frac{1}{n^{2} \Gamma}\right),
$$

which is an ellipse in $\left\{\beta_{z}, \beta_{\perp}\right\}$ space with origin at $\beta_{z}=$ $1 /(n \Gamma), \beta_{\perp}=0$. Equation (15) shows that if $n \gg 1$, then $\varepsilon>1$ in which case $\varepsilon^{2} \gg 1 / n^{2}$ and so the coefficient of $\beta_{\perp}^{2}$ in Eq. (16) becomes unity and the ellipse becomes a circle with center offset to the right from the origin. Equation (16) can solved for $\beta_{\perp}$ to obtain

$$
\beta_{\perp}= \pm \frac{1}{\varepsilon} \sqrt{\varepsilon^{2}-1+\frac{1}{n^{2} \Gamma}-\frac{1}{\Gamma}\left(\Gamma \beta_{z}-\frac{1}{n}\right)^{2}} .
$$

The allowed maximum and minimum values of $\beta_{z}$ are found by setting $\beta_{\perp}=0$ in Eq. (16) to give

$$
\begin{aligned}
& \beta_{z \min }=\frac{1}{n \Gamma}-\sqrt{\frac{\varepsilon^{2}-1}{\Gamma}+\frac{1}{n^{2} \Gamma^{2}}}, \\
& \beta_{z \max }=\frac{1}{n \Gamma}+\sqrt{\frac{\varepsilon^{2}-1}{\Gamma}+\frac{1}{n^{2} \Gamma^{2}}} .
\end{aligned}
$$

The motion of the particle in the $\left\{\beta_{z}, \beta_{\perp}\right\}$ plane is constrained to lie on the ellipse defined by Eqs. (16)-(19) with $\varepsilon$ defined by the values of $\left\{\beta_{z}, \beta_{\perp}\right\}$ at $t=0$ using Eq. (15). The dotted approximate semi-ellipses in the (a) parts of Figs. 1-7 are plots of Eq. (17); the short solid line segments superimposed on these curves are numerically calculated loci of $\beta_{z}$ versus $\beta_{\perp}$ for various initial conditions. It will be shown below that the properties of these loci show a strong dependence on an angle $\phi_{0}$ relating the directions of the initial particle perpendicular velocity and the initial wave magnetic field. Figures 1-4 differ only in choice of $\phi_{0}$. Figures 5 and 6 have the same $\phi_{0}$ as Figs. 1 and 2, respectively, but a parameter related to frequency mismatch differs slightly. Figure 7 has both different $\phi_{0}$ and different mismatch compared to Fig. 1.

\section{Equation of motion in the wave frame}

Because $\gamma^{\prime}$ is constant, it may be factored from Eq. (11), which then simplifies to 

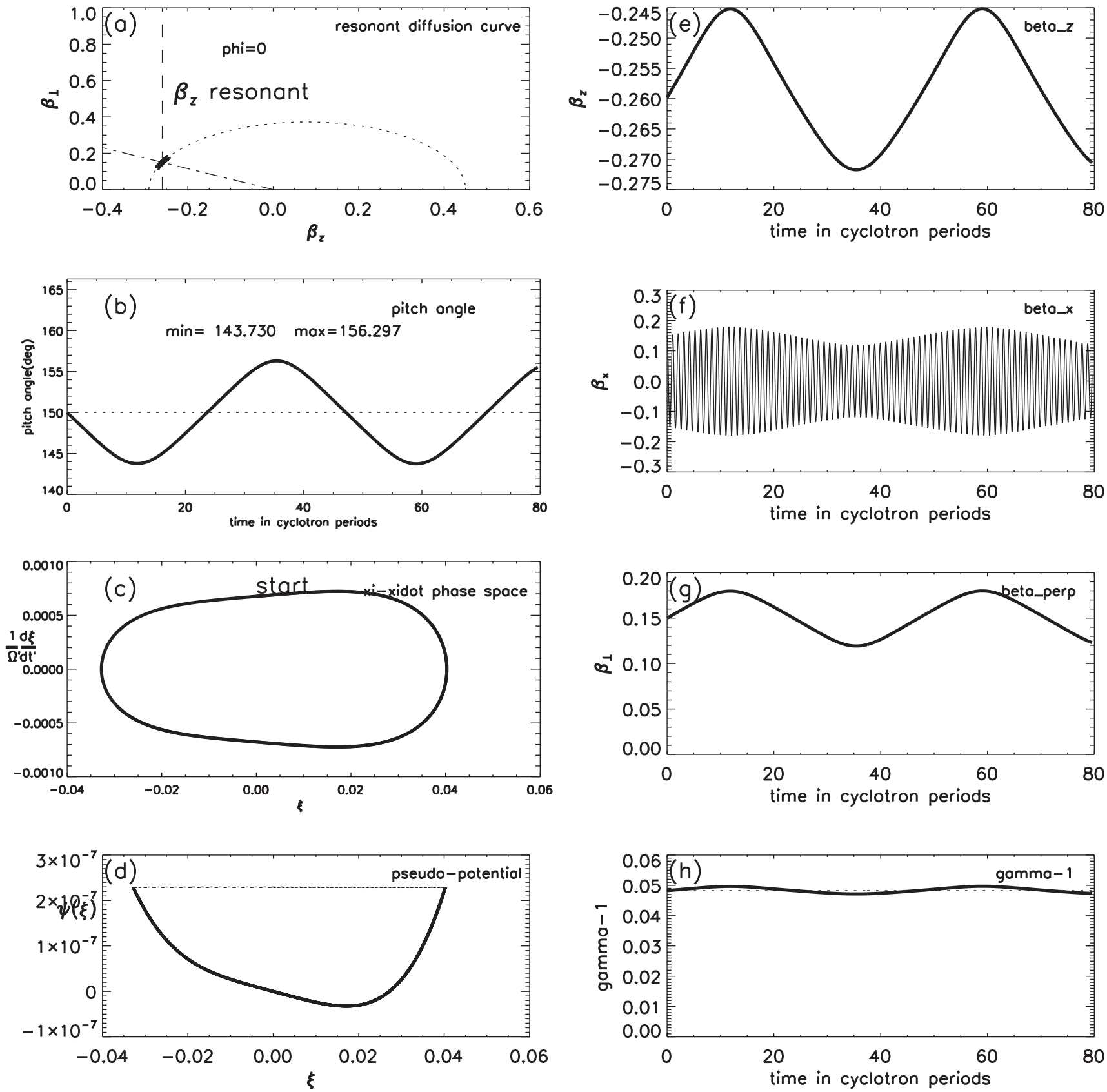

FIG. 1. Comparison between numerical integration of Eq. (4) and predictions of analytic model: Assumed input parameters are $\kappa=0.0016, \alpha=0.25$, initial pitch angle $=150^{\circ}$, initial $\beta=0.3, \phi_{0}=0$, and $\xi_{0}=0$. These give $n=10.8378$ from Eq. (33), $\gamma_{T}=1.00428$ from Eq. (9), $\gamma^{\prime}=1.07801$ from Eq. (14), $\varepsilon=$ 1.07341 from Eq. (15), $\sigma=2.90838$ from Eq. (27), and $s=0.424226$ from Eq. (39). (a) Dotted semi-ellipse shows resonant diffusion curve given by Eq. (17) with upper and lower $\beta_{z}$ limits given by Eqs. (18) and (19). The dashed vertical line shows the value of $\beta_{z}$ for which $\xi=0$. The dot-dash slanted line shows the initial pitch angle. The initial position of the particle is at the intersection of the three curves. Short solid line superimposed on resonant diffusion curve is numerically calculated locus of $\left\{\beta_{2}, \beta_{\perp}\right\}$ over 80 cyclotron periods. (b) Pitch angle versus time with initial pitch angle shown for reference as horizontal dotted line. (c) Phase-space plot of $\left(\Omega^{\prime}\right)^{-1} d \xi / d t^{\prime}$ versus $\xi$ over 80 cyclotron periods. Starting position shown, orbit is clockwise. (d) Plot of $\psi(\xi)$ as determined from Eq. (41). Dashed line is left hand side of Eq. (42), where $\xi$ is determined from instantaneously numerically calculated $\beta_{z}$ using Eqs. (13) and (29) and where $d \xi / d t^{\prime}$ is determined from Eq. (45) using instantaneous numerically calculated $\boldsymbol{\beta}$. Flatness of dashed line shows that pseudo-energy is conserved. (e) Plot of numerically calculated $\beta_{z}$; note that moving to more negative values on curve corresponds to moving towards loss cone. (f) Plot of numerically calculated $\beta_{x} ; \beta_{y}$ is not shown but is essentially similar but approximately in quadrature. (g) Plot of numerically calculated $\beta_{\perp}$. (h) Plot of numerically calculated $\gamma-1$, which in the non-relativistic limit corresponds to the usual kinetic energy.

$$
\frac{1}{\Omega^{\prime}} \frac{d \beta^{\prime}}{d t^{\prime}}=\beta^{\prime} \times\left(\hat{z}+\frac{\tilde{\mathbf{B}}_{\perp}^{\prime}}{B_{0}}\right),
$$

where $\Omega^{\prime}=\Omega / \gamma^{\prime}$ is the particle's cyclotron frequency as observed in the wave frame. It should be noted that $\Omega^{\prime}$ is constant because $\gamma^{\prime}$ is constant. Equation (20) can be decomposed into parallel and perpendicular components

$$
\frac{1}{\Omega^{\prime}} \frac{d \beta_{z}^{\prime}}{d t^{\prime}}=\hat{z} \cdot\left(\beta_{\perp}^{\prime} \times \frac{\tilde{\mathbf{B}}_{\perp}^{\prime}}{B_{0}}\right),
$$



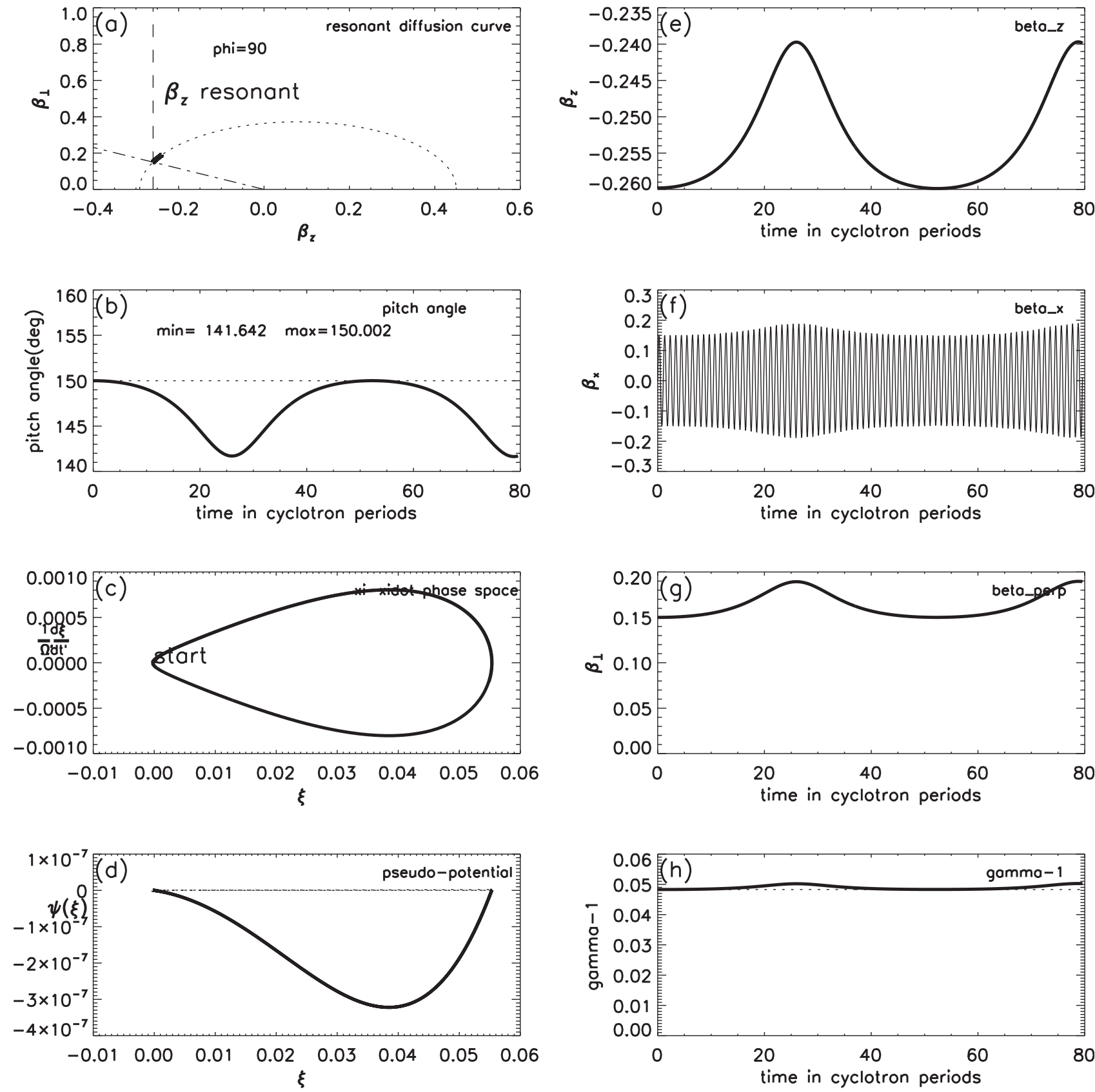

FIG. 2. Same as Fig. 1 except now $\phi_{0}=90^{\circ}$.

$$
\overline{\Omega^{\prime}} \frac{d \beta_{\perp}^{\prime}}{d t^{\prime}}=\beta_{\perp}^{\prime} \times \hat{z}+\beta_{z}^{\prime} \hat{z} \times \frac{\tilde{\mathbf{B}}_{\perp}^{\prime}}{B_{0}} .
$$

\section{E. Construction of a pseudo-potential problem}

Taking the derivative of Eq. (21) with respect to $t^{\prime}$ gives

$$
\frac{1}{\Omega^{\prime}} \frac{d^{2} \beta_{z}^{\prime}}{d t^{\prime 2}}=\hat{z} \cdot\left(\frac{d \beta_{\perp}^{\prime}}{d t^{\prime}} \times \frac{\mathbf{B}_{\perp}^{\prime}}{B_{0}}\right)+\hat{z} \cdot\left(\beta_{\perp}^{\prime} \times \frac{d}{d t^{\prime}}\left(\frac{\mathbf{B}_{\perp}^{\prime}}{B_{0}}\right)\right) .
$$

Using Eq. (3) in Eqs. (8) and (10) for the phase, it is seen that the perpendicular magnetic field in the wave frame is

$$
\begin{aligned}
\mathbf{B}_{\perp}^{\prime} & =\gamma_{T}\left(\mathbf{B}-\frac{1}{c^{2}} \frac{\omega}{k} \hat{z} \times\left(-\frac{\omega}{k} \hat{z} \times \tilde{\mathbf{B}}\right)\right)_{\perp} \\
& =-\hat{x} \tilde{B}^{\prime} \sin \left(k^{\prime} z^{\prime}\right)+\hat{y} \tilde{B}^{\prime} \cos \left(k^{\prime} z^{\prime}\right),
\end{aligned}
$$

where $\tilde{B}^{\prime} / B_{0}=\kappa^{\prime}=\kappa / \gamma_{T}$. The wave-frame time derivative of the magnetic field is

$$
\begin{aligned}
\frac{d \tilde{\mathbf{B}}_{\perp}^{\prime}}{d t^{\prime}} & =-k^{\prime} \frac{d z^{\prime}}{d t^{\prime}} \tilde{B}^{\prime}\left(\hat{x} \cos \left(k^{\prime} z^{\prime}\right)+\hat{y} \sin \left(k^{\prime} z^{\prime}\right)\right) \\
& =\omega n^{\prime} \beta_{z}^{\prime} \hat{z} \times \tilde{\mathbf{B}}_{\perp}^{\prime},
\end{aligned}
$$

where $n^{\prime}=c k^{\prime} / \omega=n / \gamma_{T}$. Substitution using Eqs. (22) and (25) into Eq. (23) gives

$$
\frac{1}{\Omega^{\prime 2}} \frac{d^{2} \beta_{z}^{\prime}}{d t^{\prime 2}}=\left(1+\sigma \beta_{z}^{\prime}\right) \beta_{\perp}^{\prime} \cdot \frac{\tilde{\mathbf{B}}_{\perp}^{\prime}}{B_{0}}-\beta_{z}^{\prime} \frac{\tilde{\mathbf{B}}_{\perp}^{\prime}}{B_{0}} \cdot \frac{\tilde{\mathbf{B}}_{\perp}^{\prime}}{B_{0}},
$$

where

$$
\sigma=\frac{\omega}{\Omega^{\prime}} n^{\prime}=\frac{\omega}{\Omega} \frac{\gamma^{\prime}}{\gamma_{T}} n=\varepsilon \alpha n
$$



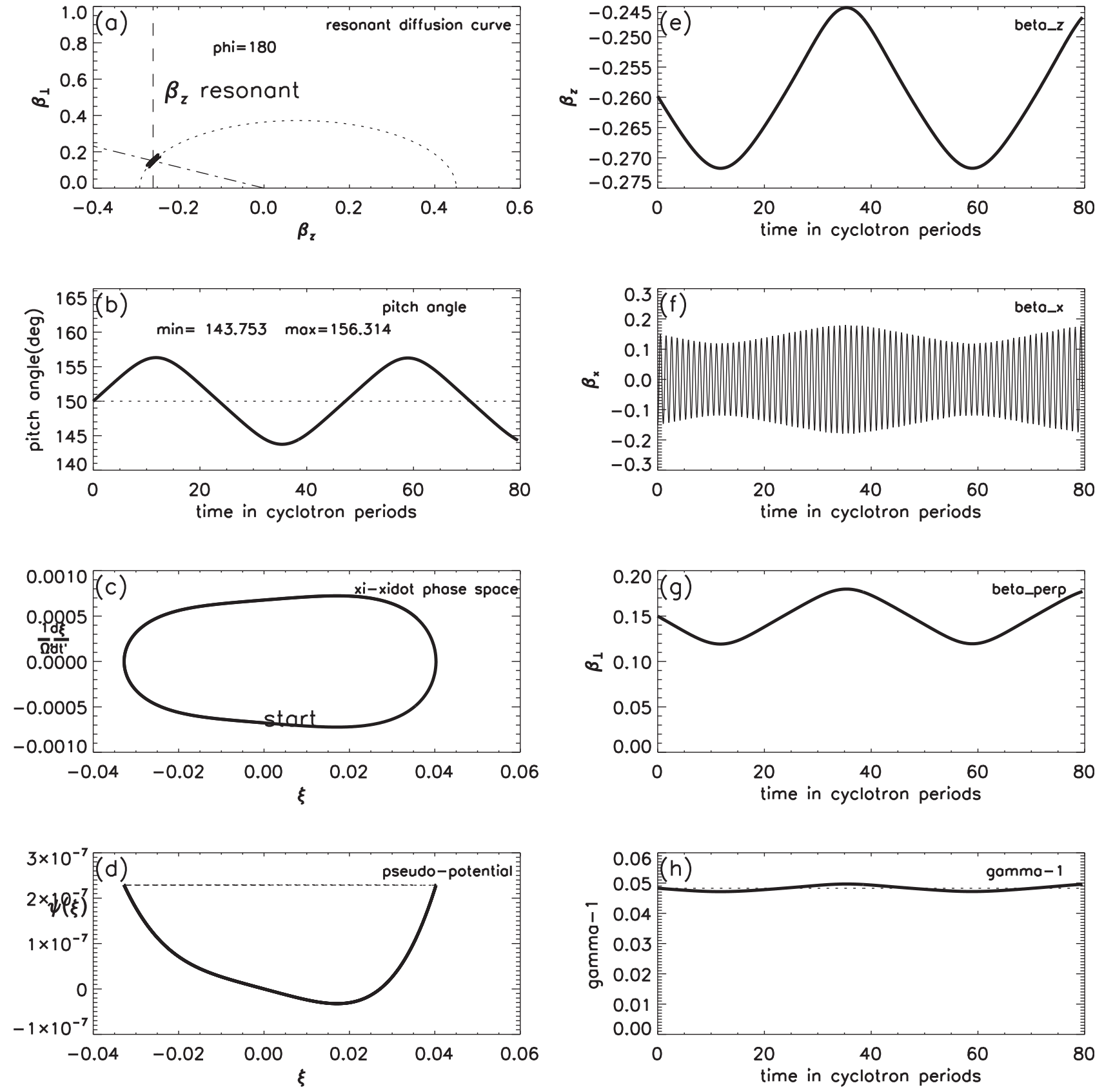

FIG. 3. Same as Fig. 1 except now $\phi_{0}=180^{\circ}$.

is a constant fixed by initial conditions.

The last term on the right hand side of Eq. (26) is linear in $\beta_{z}^{\prime}$ since $\tilde{\mathbf{B}}_{\perp}^{\prime} \cdot \tilde{\mathbf{B}}_{\perp}^{\prime}$ is constant, so if this last term were the only term on the right hand side, Eq. (26) would be an oscillator equation with $\beta_{z}^{\prime}$ as the oscillating variable. The only quantity on the right hand side of Eq. (26), which is not an obvious explicit function of $\beta_{z}^{\prime}$ is $\beta_{\perp}^{\prime} \cdot \tilde{\mathbf{B}}_{\perp}^{\prime} / B_{0}$, but we will now show that $\beta_{\perp}^{\prime} \cdot \tilde{\mathbf{B}}_{\perp}^{\prime} / B_{0}$ can in fact be expressed a function of $\beta_{z}^{\prime}$. To obtain such an expression, it is first noted that inserting Eqs. (22) and (25) into the time derivative of $\beta_{\perp}^{\prime} \cdot \tilde{\mathbf{B}}_{\perp}^{\prime} / B_{0}$ gives

$$
\begin{aligned}
\frac{d}{d t^{\prime}}\left(\beta_{\perp}^{\prime} \cdot \frac{\tilde{\mathbf{B}}_{\perp}^{\prime}}{B_{0}}\right) & =-\hat{z} \cdot \beta_{\perp}^{\prime} \times \frac{\tilde{\mathbf{B}}_{\perp}^{\prime}}{B_{0}}\left(\Omega^{\prime}+\omega n^{\prime} \beta_{z}^{\prime}\right) \\
& =-\left(1+\sigma \beta_{z}^{\prime}\right) \frac{d \beta_{z}^{\prime}}{d t^{\prime}} .
\end{aligned}
$$

At this point, it is useful to define the frequency mismatch

$$
\xi=1+\sigma \beta_{z}^{\prime}
$$

Since $\xi$ is a linear function of $\beta_{z}^{\prime}$, it can be considered to be a proxy for $\beta_{z}^{\prime}$ that is more convenient to use than $\beta_{z}^{\prime}$ itself. By writing $\xi$ in terms of unprimed quantities, it is seen that

$$
\xi=1+\alpha \gamma\left(n \beta_{z}-1\right),
$$

so the initial value of $\xi$ defines the following situations of special interest:

(1) Resonant particle. This situation has $\xi_{0}=0$, so Eq. (30) becomes 

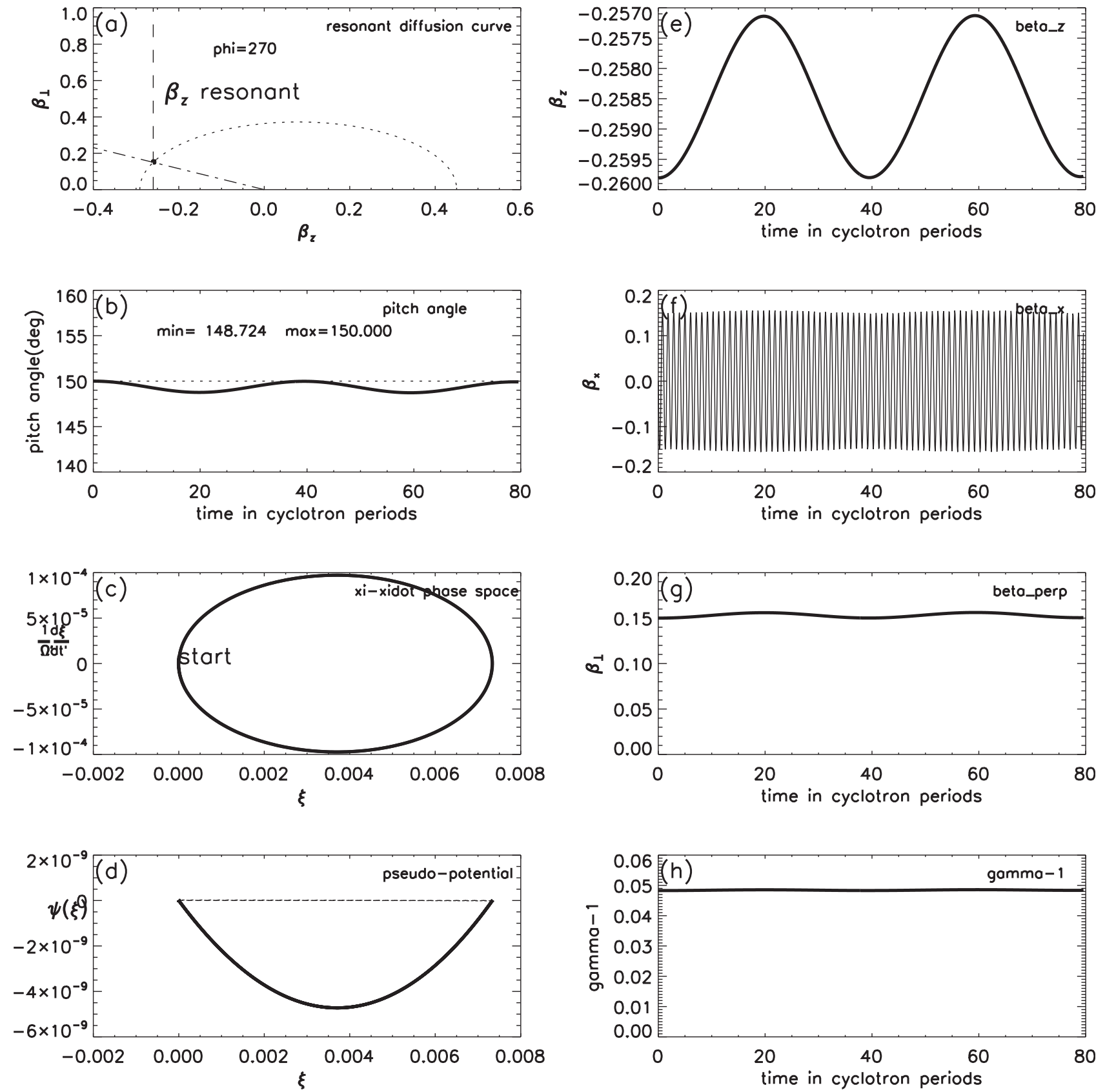

FIG. 4. Same as Fig. 1 except now $\phi_{0}=270^{\circ}$. Note that behavior differs from $90^{\circ}$.

$$
\omega-k v_{z 0}=\frac{\Omega}{\gamma_{0}},
$$

which is the initial condition for Eq. (1).

Equation (31) can be written as

$$
\beta_{z 0}=-\frac{1}{n}\left(\frac{1}{\alpha \gamma_{0}}-1\right),
$$

which shows that if $\omega<\Omega / \gamma_{0}, \beta_{z 0}$ is negative. The dashed vertical lines in the (a) parts of Figs. 1-7 show $\beta_{z 0}$ given by Eq. (32). The intersection of these dashed vertical lines with the dotted semi-circle resonance diffusion curve gives the starting position in $\left\{\beta_{z}, \beta_{\perp}\right\}$ space of a resonant particle. The sloped dashed-dotted line indicates the initial pitch angle of this resonant particle.
(2) Particle initially at rest in the wave frame (i.e., $\left.\beta_{z}^{\prime}=0\right)$. This situation has $\xi_{0}=1$ and $v_{z 0}=\omega / k$.

(3) Particle initially at rest in the lab frame. This situation has $\xi_{0}=1-\alpha$ since $\gamma_{0}=1$ for a particle initially at rest.

Thus, $\xi$ is indeed a measure of the frequency mismatch as experienced by the particle. It is sometimes convenient to specify $\xi$ and then solve for $n$, i.e., specify the mismatch for a given $\beta_{z}, \gamma$, and $\alpha$, so that $n$ is given by

$$
n=\frac{1}{\beta_{z}}\left(1+\frac{\xi-1}{\alpha \gamma}\right) .
$$

Returning to the construction of an oscillator equation, it is seen that $d \xi / d t^{\prime}=\sigma d \beta_{z}^{\prime} / d t^{\prime}$, so Eq. (28) becomes 

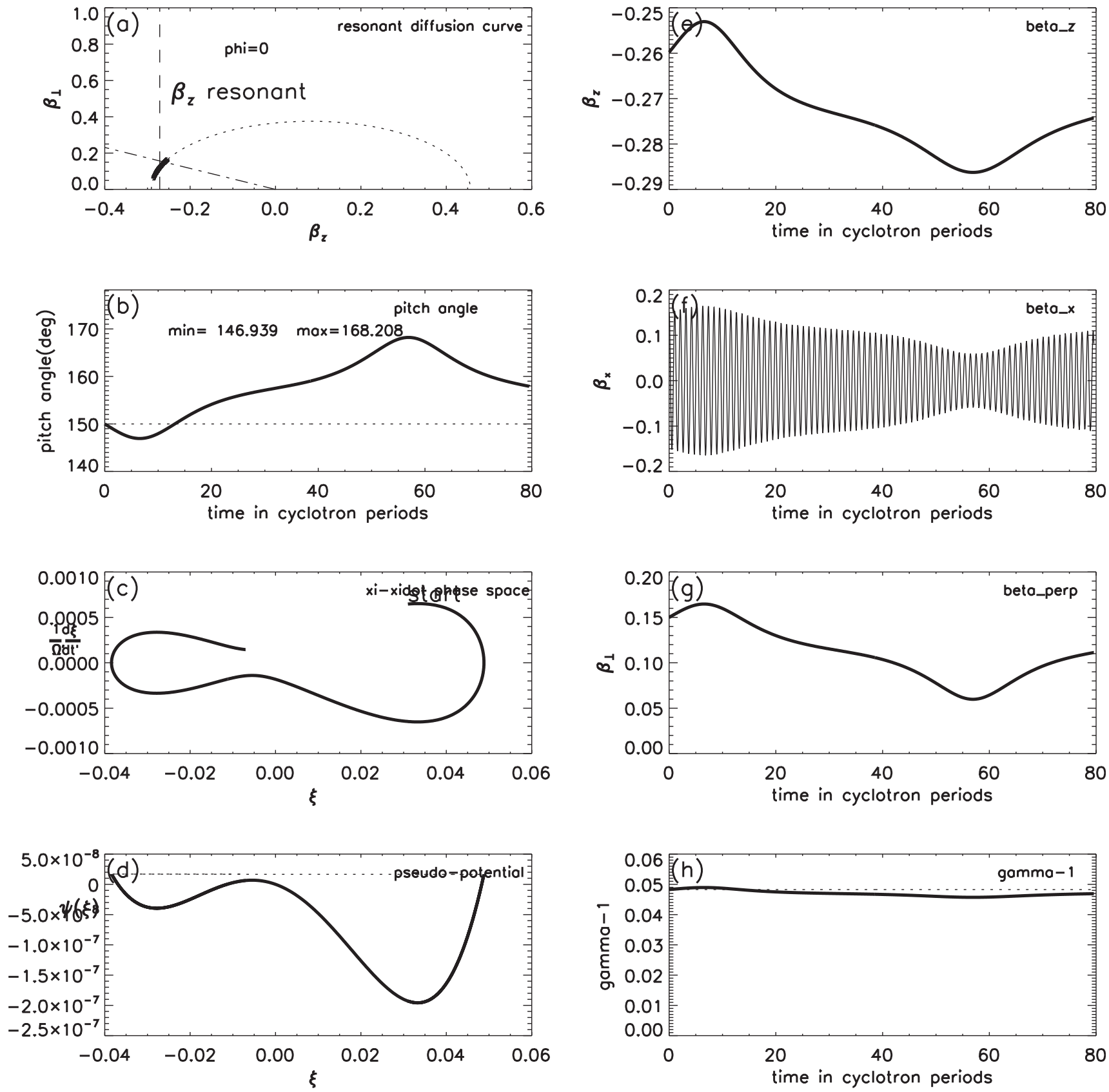

FIG. 5. Same as Fig. 1 except now $\xi_{0}=0.031$. Comparison between numerical integration of Eq. (4) and predictions of analytic model. The slight change in $\xi_{0}$ results in slight changes in some of the derived parameters: in particular, now $n=10.3826, \varepsilon=1.07452, \sigma=2.78906, s=0.406248$, $\gamma_{T}=1.00467$, and $\gamma^{\prime}=1.07954$. Note extreme excursion to negative $\xi$ as particle enters left hand valley in (d) and that this gives an extreme jump in pitch angle to over $168^{\circ}$.

$$
\frac{d}{d t^{\prime}}\left(\beta_{\perp}^{\prime} \cdot \frac{\tilde{\mathbf{B}}_{\perp}^{\prime}}{B_{0}}\right)=-\frac{1}{2 \sigma} \frac{d \xi^{2}}{d t^{\prime}},
$$

which can be integrated to give

$$
\beta_{\perp}^{\prime} \cdot \frac{\tilde{\mathbf{B}}_{\perp}^{\prime}}{B_{0}}=\beta_{\perp 0}^{\prime} \cdot \frac{\tilde{\mathbf{B}}_{\perp 0}^{\prime}}{B_{0}}-\frac{1}{2 \sigma}\left(\xi^{2}-\xi_{0}^{2}\right) .
$$

Noting that $t=0$ corresponds to $t^{\prime}=0$ because $z=0$ at $t=0$ and recalling that $\gamma^{\prime}$ is constant, it is seen from Eq. (12) that

$$
\gamma^{\prime} \beta_{\perp 0}^{\prime}=\gamma_{0} \beta_{\perp 0} .
$$

Since $\tilde{\mathbf{B}}_{\perp 0}^{\prime}$ is in the $y$ direction when $z^{\prime}=0$, it is seen that

$$
\beta_{\perp 0}^{\prime} \cdot \frac{\tilde{\mathbf{B}}_{\perp 0}^{\prime}}{B_{0}}=\beta_{y 0}^{\prime} \frac{\tilde{B}^{\prime}}{B_{0}}=\frac{\gamma_{0}}{\gamma^{\prime}} \kappa^{\prime} \beta_{y 0}=\frac{\gamma_{0}}{\gamma^{\prime}} \kappa^{\prime} \beta_{\perp 0} \sin \phi_{0},
$$

where $\phi_{0}$ is defined by $\beta_{x 0}=\beta_{\perp 0} \cos \phi_{0}$ and $\beta_{y 0}=\beta_{\perp 0} \sin \phi_{0}$. Thus, $\phi_{0}=0$ and $\phi_{0}=\pi$ correspond to the initial velocity being orthogonal to the initial wave magnetic field. With these definitions, Eq. (35) becomes

$$
\beta_{\perp}^{\prime} \cdot \frac{\tilde{\mathbf{B}}_{\perp}^{\prime}}{B_{0}}=\frac{\gamma_{0}}{\gamma^{\prime}} \kappa^{\prime} \beta_{\perp 0} \sin \phi_{0}-\frac{1}{2 \sigma}\left(\xi^{2}-\xi_{0}^{2}\right) .
$$



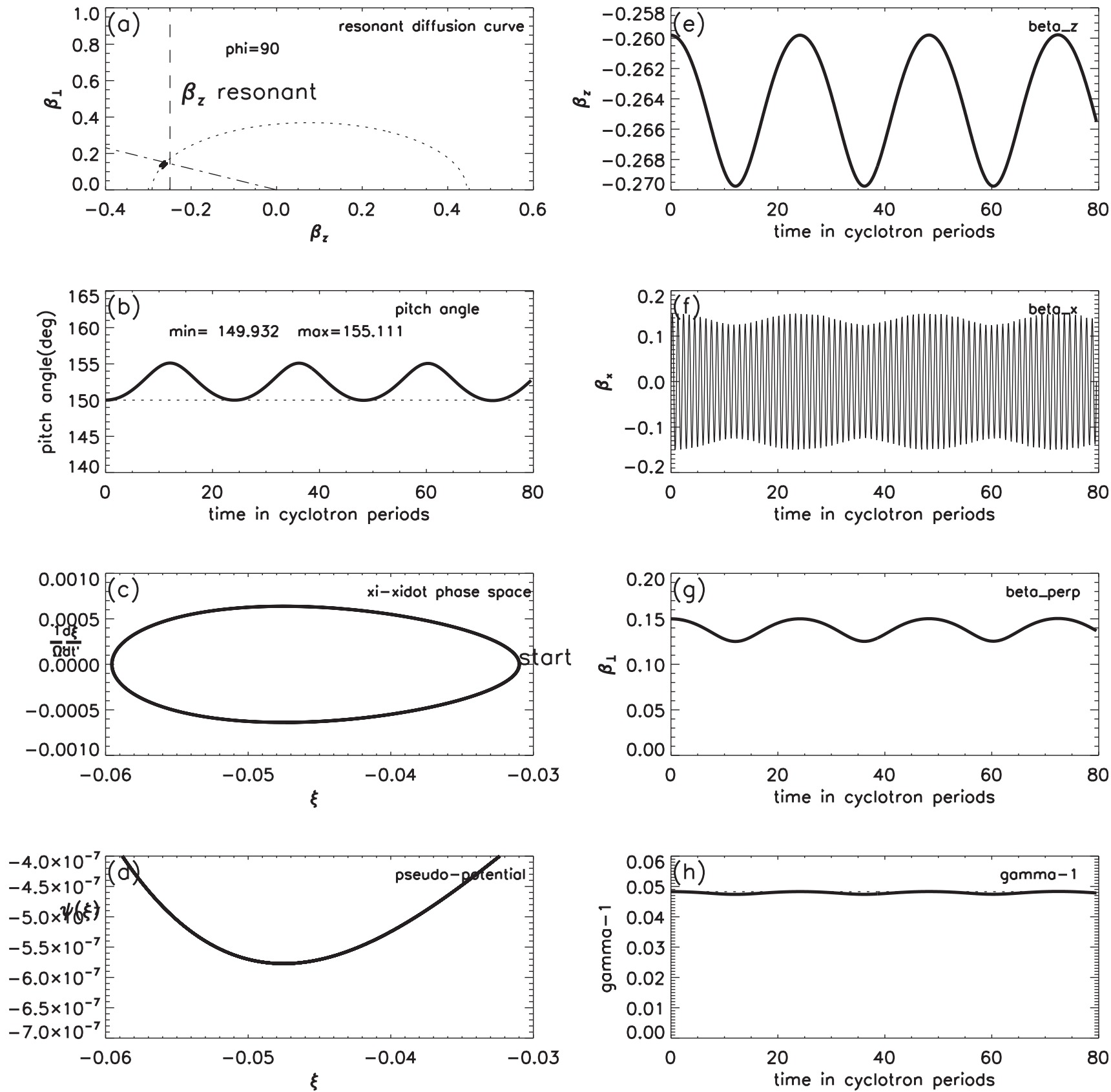

FIG. 6. Same as Fig. 2, except $\xi_{0}=-0.031$. The slight change in $\xi_{0}$ causes slight changes in derived parameters, which are now $n=11.2931, \varepsilon=1.07420, \sigma=3.02770, s=0.442197, \gamma_{T}=1.00394$, and $\gamma^{\prime}=1.07663$.

On defining

$$
s=\alpha n \beta_{\perp 0} \gamma_{0} / \gamma_{T}
$$

as a constant determined by initial conditions, and then using Eqs. (29) and (38), Eq. (26) can be expressed as an equation of motion for a pseudo-particle moving in $\xi$ space,

$$
\frac{1}{\Omega^{\prime 2}} \frac{d^{2} \xi}{d t^{\prime 2}}=-\frac{\partial \psi}{\partial \xi}
$$

where

$$
\psi(\xi)=\frac{1}{8} \xi^{4}+\left(\kappa^{\prime 2}-\frac{\xi_{0}^{2}}{2}-s \kappa^{\prime} \sin \phi_{0}\right) \frac{\xi^{2}}{2}-\kappa^{\prime 2} \xi
$$

is a pseudo-potential characterized by the four constants $\xi_{0}$, $\kappa^{\prime}, s$, and $\phi_{0}$.

Multiplying Eq. (40) by $d \xi / d t^{\prime}$ and integrating gives a pseudo-energy equation

$$
\frac{1}{2 \Omega^{\prime 2}}\left(\frac{d \xi}{d t^{\prime}}\right)^{2}+\psi(\xi)=W
$$

where

$$
W=\frac{1}{2 \Omega^{\prime 2}}\left(\frac{d \xi}{d t^{\prime}}\right)_{0}^{2}+\psi\left(\xi_{0}\right)=\text { const } .
$$

is the total pseudo-energy, i.e., the sum of the pseudo-kinetic and pseudo-potential energies. 

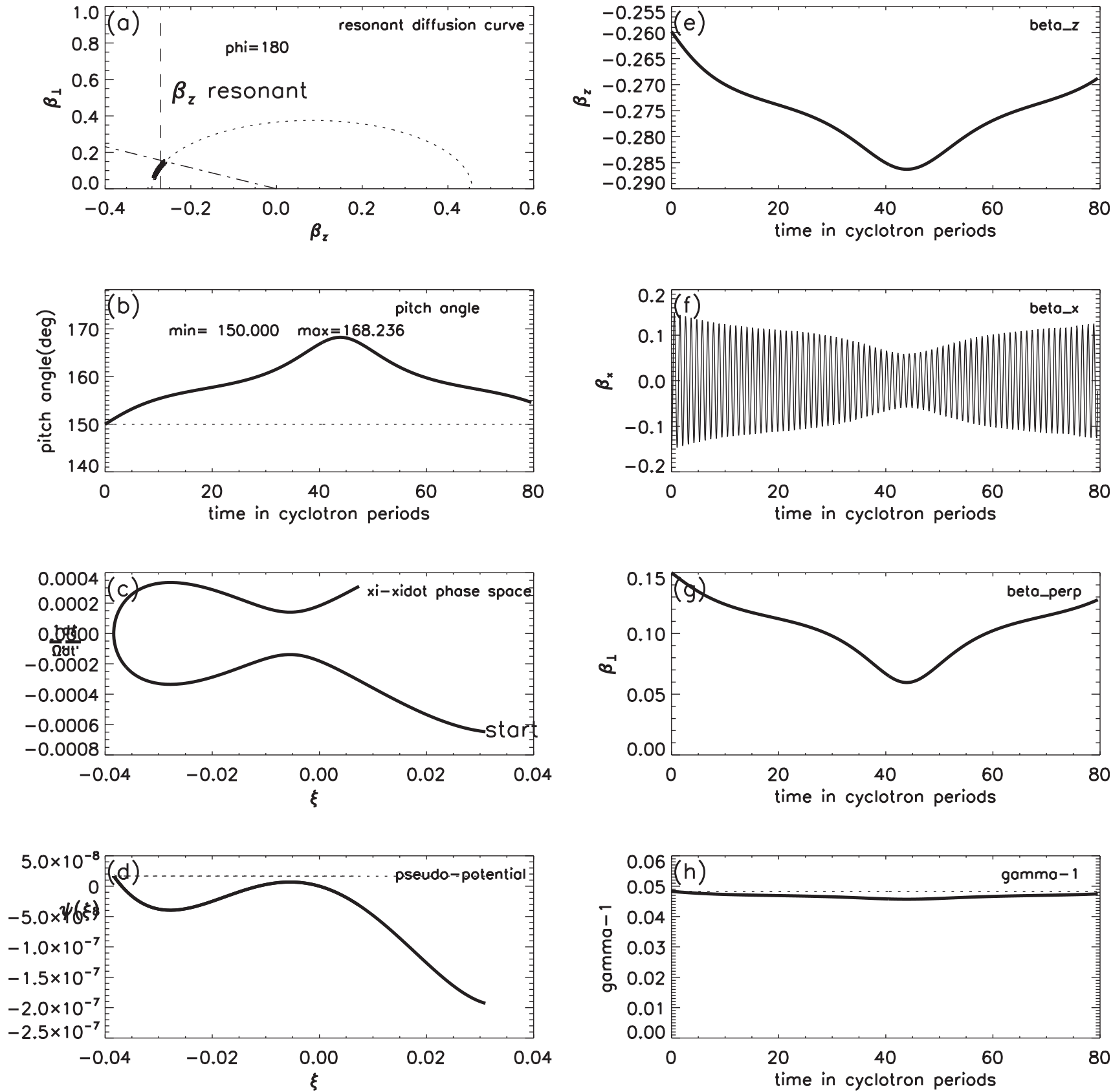

FIG. 7. Same as Fig. 5 (i.e., $\xi_{0}=0.031$ ) except now $\phi_{0}=180^{\circ}$. Pseudo-particle in same two-valley potential as Fig. $5 \operatorname{since} \sin \phi_{0}=0$ for both $\phi_{0}=0$ and $\phi_{0}=\pi$, but now starts with negative $d \xi / d t \sim \cos \phi_{0}$ and so pitch angle starts increasing immediately.

Equation (42) shows that the motion in the wave frame has the form of an anharmonic oscillator, where $\left(1 / \Omega^{\prime}\right) d \xi / d t^{\prime}$ plays the role of velocity and $\xi$ plays the role of position. Figure 8 shows that $\psi(\xi)$ has one of two characteristic profiles depending on the sign of the coefficient of $\xi^{2}$ in Eq. (41). If as in Figs. 8(a) and 8(b), the coefficient of $\xi^{2}$ is positive then $\psi(\xi)$ has a single minimum (valley), which is to the right of $\xi=0$ whereas if as in Figs. 8(c) and 8(d), the coefficient of $\xi^{2}$ is negative, there are two minima (valleys), one to the left of $\xi=0$ and one to the right. Figure 8(a) sketches the individual behavior of the terms $\sim \xi^{4}, \sim \xi^{2}$, and $\sim-\xi$ while Fig. 8(b) sketches the sum of these terms, i.e., the behavior of $\psi(\xi)$ when the coefficient of $\xi^{2}$ is positive. Figure 8(c) sketches the individual behavior of $\sim \xi^{4}$, $\sim-\xi^{2}$, and $\sim-\xi$ while Fig. 8(d) sketches the sum of these terms, i.e., the behavior of $\psi(\xi)$ when the coefficient of $\xi^{2}$ is negative. The $-\xi$ term provides a gradient when $\xi=0$ so a pseudo-particle starting with $\xi_{0}=0$ will always have an initial force pointing to the right because of this term (the $\xi^{4}$ and $\pm \xi^{2}$ terms have zero gradient at $\xi=0$ ). Whether the potential is of the form shown in Fig. 8(b) and 8(d) has a profound effect on the actual particle motion; this will be discussed in more detail below. The motion is also significantly affected by the magnitude of the initial $d \xi / d t^{\prime}$ because this affects the total pseudo-energy, which is the sum of the pseudo-potential and the pseudo-kinetic energies. If $\left|d \xi / d t^{\prime}\right|$ is initially zero, the pseudo-particle cannot access $\xi$ regions where $\psi(\xi)$ exceeds $\psi\left(\xi_{0}\right)$ but if $\left|d \xi / d t^{\prime}\right|$ is initially finite, the pseudo-particle may access certain $\xi$ regions where $\psi(\xi)$ exceeds $\psi\left(\xi_{0}\right)$. Also, if $d \xi / d t^{\prime}$ is initially negative, then the 

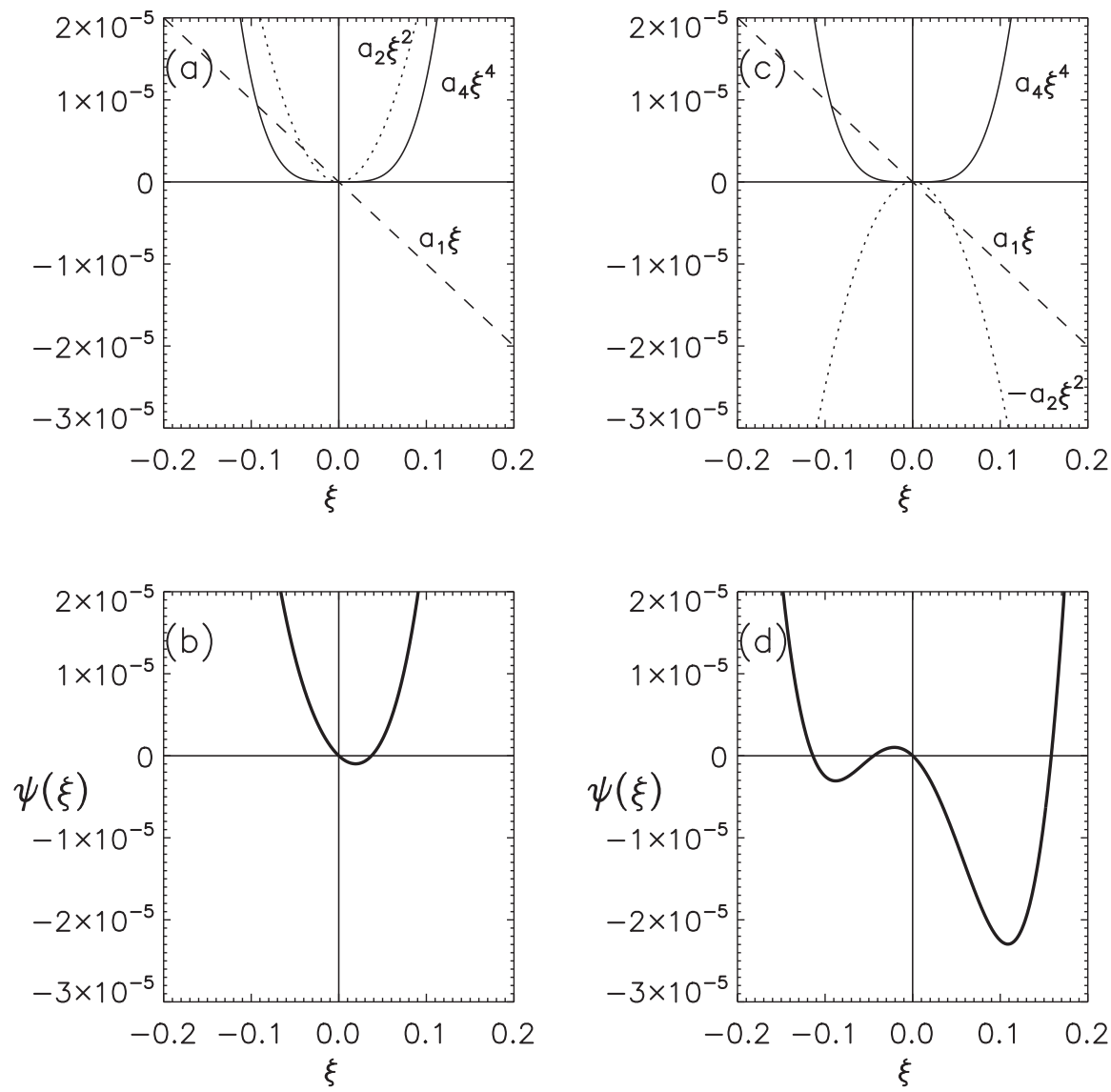

FIG. 8. Demonstration showing how there is one shallow valley if coefficient of $\xi^{2}$ in $\psi(\xi)$ is positive, and two larger valleys if coefficient of $\xi^{2}$ is negative. Coefficients used here are $a_{4}=0.125$ $a_{2}=0.0025$, and $a_{1}=-0.0001$. (a) Individual plots of $a_{4} \xi^{4}, a_{2} \xi^{2}$, and $a_{1} \xi$. (b) Plot where $\psi(\xi)=a_{4} \xi^{4}+a_{2} \xi^{2}+a_{1} \xi$. (c) Individual plots of $a_{4} \xi^{4},-a_{2} \xi^{2}$, and $a_{1} \xi$. (d) Plot where $\psi(\xi)=a_{4} \xi^{4}$ $-a_{2} \xi^{2}+a_{1} \xi$. particle will initially move to the left even though the initial force on it is pointing to the right.

The short solid lines in the (a) parts of Figs. 1-7 show the locus of numerically integrated $\left\{\beta_{z}, \beta_{\perp}\right\}$, where $\beta_{\perp}=\sqrt{\beta_{x}^{2}+\beta_{y}^{2}}$. The values of $\beta$, initial pitch angle, and $\alpha$ are given in the caption of Fig. 1. Other relevant quantities are calculated from these specified independent parameters using equations listed in the caption. The solid line in the (b) parts of Figs. 1-7 show the variation in pitch angle as a function of time measured in cyclotron periods as determined by the numerical integration. The (c) parts of Figs. 1-7 present plots of $\left(1 / \Omega^{\prime}\right) d \xi / d t^{\prime}$ versus $\xi$. The particle makes clockwise orbits in this $\left\{\xi,\left(1 / \Omega^{\prime}\right) d \xi / d t^{\prime}\right\}$ phase space because $d \xi / d t^{\prime}$ is positive in the upper half plane and negative in the lower half plane.

Figures 1-4 show numerical calculations of Eq. (4) for $\phi_{0}=0, \pi / 2, \pi, 3 \pi / 2$ using $\xi_{0}=0$. Figures 5 and 6 have $\phi_{0}=0$ and $\phi_{0}=\pi / 2$, respectively, but $\xi_{0}$ is slightly different from zero (causing $n$ and $\gamma_{T}$ to be slightly different from before). Figure 7 has $\phi_{0}=\pi$ and the same finite $\xi_{0}$ as Fig. 5 . These numerical calculations will now be interpreted in terms of the particle bouncing in various shapes of pseudopotential.

From Eqs. (21) and (29), we see that

$$
\frac{1}{\Omega^{\prime}} \frac{d \xi}{d t^{\prime}}=\sigma \hat{z} \cdot\left(\beta_{\perp}^{\prime} \times \frac{\tilde{\mathbf{B}}_{\perp}^{\prime}}{B_{0}}\right),
$$

$$
\begin{aligned}
\frac{1}{\Omega^{\prime}}\left(\frac{d \xi}{d t^{\prime}}\right) & =\sigma\left(\beta_{x}^{\prime} \frac{\tilde{B}_{y}^{\prime}}{B_{0}}-\beta_{y}^{\prime} \frac{\tilde{B}_{x}^{\prime}}{B_{0}}\right) \\
& =\alpha n \frac{\gamma}{\gamma_{T}} \kappa^{\prime}\left(\beta_{x} \cos (k z-\omega t)+\beta_{y} \sin (k z-\omega t)\right)
\end{aligned}
$$

At the start where $t=0$ and $z=0$,

$$
\frac{1}{\Omega^{\prime}}\left(\frac{d \xi}{d t^{\prime}}\right)_{t^{\prime}=t=0}=s \kappa^{\prime} \cos \phi_{0} .
$$

The (d) parts of Figs. 1-7 plot $\psi(\xi)$ (solid line). To prove that pseudo-energy is conserved, the two terms on the left hand side of Eq. (42) are individually computed from the numerical calculation of the motion and the sum of these two terms is plotted as the dotted line in these (d) parts. Specifically, at each time during the calculation, the instantaneous value of the potential term $\psi(\xi)$ is determined using numerically calculated $\beta_{x}, \beta_{y}$, and $\beta_{z}$ transformed into an instantaneous $\xi$ using Eq. (30). The "kinetic" term $\left(2 \Omega^{\prime 2}\right)^{-1}\left(d \xi / d t^{\prime}\right)^{2}$ is determined using the numerically calculated $\beta_{x}, \beta_{y}$, and $\beta_{z}$ in Eq. (45) to evaluate $\left(1 / \Omega^{\prime}\right) d \xi / d t^{\prime}$. The fact that the dashed line is flat in the $(\mathrm{d})$ parts of Figs. 1-7 confirms the anharmonic oscillatory nature of the motion and shows that the pseudo-energy is conserved.

\section{F. Turning points of bounce motion in pseudo-potential well}

Combined numerical and analytical investigation show that the motion of the pseudo-particle in the pseudo-potential 
well has a number of distinct behaviors, which we classify into (a) two types of ordinary motion in a single valley, which is either completely in positive $\xi$ space or can straddle positive and negative $\xi$ space, (b) a rather spectacular motion involving going back and forth between two valleys and giving an extreme pitch angle change, (c) a less spectacular but nevertheless distinct motion in negative $\xi$ space, and (d) motion far from resonance.

\section{Ordinary motion}

Because $\psi(\xi)$ behaves as a potential well for an anharmonic oscillator, it is seen from Eq. (42) that $d \xi / d t^{\prime}$ vanishes at the maximum particle excursions in this potential well. These turning points are, therefore, defined by the condition

$$
\psi\left(\xi_{t p}\right)=\frac{1}{2 \Omega^{\prime 2}}\left(\frac{d \xi}{d t^{\prime}}\right)_{0}^{2}+\psi\left(\xi_{0}\right) .
$$

Using Eqs. (41) and (46), this becomes

$$
\begin{aligned}
& \frac{\xi_{t p}^{4}}{8}-\left(\frac{\xi_{0}^{2}}{2}+s \kappa^{\prime} \sin \phi_{0}-\kappa^{\prime 2}\right) \frac{\xi_{t p}^{2}}{2}-\kappa^{\prime 2} \xi_{t p} \\
& =\frac{s^{2}}{2} \kappa^{\prime 2} \cos ^{2} \phi_{0}+\frac{\xi_{0}^{4}}{8}-\left(\frac{\xi_{0}^{2}}{2}+s \kappa^{\prime} \sin \phi_{0}-\kappa^{\prime 2}\right) \frac{\xi_{0}^{2}}{2}-\kappa^{\prime 2} \xi_{0} .
\end{aligned}
$$

This shows that if $\phi_{0}= \pm \pi / 2$ so that $d \xi / d t^{\prime}=0$ at $t=0$, then $\xi_{0}$ is one of the turning points whereas if $\phi_{0} \neq \pm \pi / 2$, then $\xi_{0}$ is not a turning point in which case the particle will move on both sides of $\xi_{0}$.

Let us now consider the situation of a resonant particle (see Eq. (31)), i.e., a particle that has $\xi_{0}=0$. In this situation, Eq. (48) becomes

$$
\frac{1}{8} \xi_{t p}^{4}-\left(s \kappa^{\prime} \sin \phi_{0}-\kappa^{\prime 2}\right) \frac{\xi_{t p}^{2}}{2}-\kappa^{\prime 2} \xi_{t p}=s^{2} \kappa^{\prime 2} \cos ^{2} \phi_{0} .
$$

If $\phi_{0}=\pi / 2$, this reduces to

$$
\frac{1}{8} \xi_{t p}^{4}-\left(s \kappa^{\prime}-\kappa^{\prime 2}\right) \frac{\xi_{t p}^{2}}{2}-\kappa^{\prime 2} \xi_{t p}=0 .
$$

Since $\xi_{0}=0$ is a turning point and since the particle moves to the right initially, the other turning point must be at positive $\xi$. Assuming that the amplitude of the wave magnetic field is very small compared to the background magnetic field, we assume that $\kappa^{\prime} \ll 1$, so Eq. (50) has the nonnegative roots

$$
\xi_{t p}=0 \quad \text { and } \quad \xi_{t p} \simeq 2 \sqrt{\mathrm{s} \kappa^{\prime}}\left(1+\frac{1}{2 s^{3 / 2}} \sqrt{\kappa^{\prime}}\right) .
$$

$$
\text { If } \phi_{0}=-\pi / 2 \text {, Eq. (49) reduces to }
$$

$$
\frac{1}{8} \xi_{t p}^{4}+\left(s \kappa^{\prime}+\kappa^{\prime 2}\right) \frac{\xi_{t p}^{2}}{2}-\kappa^{\prime 2} \xi_{t p}=0,
$$

which has the non-negative roots

$$
\xi_{t p}=0 \quad \text { and } \quad \xi_{t p} \simeq \frac{2}{s} \kappa^{\prime}\left(1-\frac{\kappa^{\prime}}{s}\left(1+\frac{1}{s^{2}}\right)\right) .
$$

On the other hand, if $\phi_{0}=0$ or $\pi$, then Eq. (48) becomes

$$
\frac{1}{8} \xi_{t p}^{4}+\frac{\kappa^{2}}{\gamma_{T}^{2}} \frac{\xi_{t p}^{2}}{2}-\frac{\kappa^{2}}{\gamma_{T}^{2}} \xi_{t p}=\frac{s^{2} \kappa^{2}}{2 \gamma_{T}^{2}},
$$

which has the two roots

$$
\xi_{t p} \simeq \pm \frac{\left(4 \kappa^{\prime 2} s^{2}\right)^{1 / 4}}{1 \mp\left(4 \kappa^{\prime 2} s^{2}\right)^{1 / 4} /\left(2 s^{2}\right)} .
$$

Let us now relate $s$ to the initial pitch angle for the case $\xi_{0}=$ 0 . In this case, Eq. (33) gives $n=\left(1-1 /\left(\alpha \gamma_{0}\right)\right) / \beta_{z 0}$, so

$$
s=-\frac{1}{\gamma_{T}} \frac{\beta_{\perp 0}}{\beta_{z 0}}\left(1-\alpha \gamma_{0}\right) .
$$

Thus, except for the relativistic $\gamma$ factors, $s$ does not depend on the magnitude of the velocity, but only on the initial pitch angle and the normalized wave frequency.

We now validate the analysis by comparing predicted turning points with the turning points observed in the numerically calculated motion. For definiteness, numbers relevant to magnetospheric chorus will be used, namely: $\alpha=0.25$, $\kappa=0.0016$, and initial pitch angle $\theta=150^{\circ}$. If we consider $\beta_{0}=0.3$ then $\beta_{\perp 0}=0.15, \beta_{z 0}=-0.26, \gamma_{0}=1 / \sqrt{1-0.3^{2}}$ $=1.048, n=10.8, \gamma_{T}=1.004$, and $s=0.424$.

(1) $\phi_{0}=+\pi / 2$ case: In this case, Eq. (51) predicts turning points at $\xi=0$ and $\xi=0.056$, which is in agreement with the numerical values of the turning points seen in Figs. 2(c) and 2(d).

(2) $\phi_{0}=-\pi / 2$ case: In this case, Eq. (53) predicts turning points at $\xi=0$ and $\xi=0.0073$, which is in agreement with Figs. 4(c) and 4(d).

(3) $\phi_{0}=0$ case: Using Eq. (55), the negative turning point will be at $\xi_{t p} \simeq-0.033$ and the positive turning point at $\xi_{t p} \simeq+0.041$, which is in agreement with Figs. 1(c) and 1(d). Figure 1(b) show that the negative turning point corresponds to a pitch angle of $156^{\circ}$, so there is a change in pitch angle of $6^{\circ}$ towards the loss cone.

(4) $\phi_{0}=\pi$ case: Here, the start position is on the bottom of the phase space curve as seen in Fig. 3(c). This is in contrast to Fig. 1(c) curve where the start position is at the top of the phase space curve. The average bounce motion is the same as the $\phi_{0}=0$ case, but here $d \xi / d t$ starts negative, so the pitch angle initially increases in Fig. 3(b) in contrast to Fig. 1(b). This distinction will be shown in $\mathrm{Sec}$. III to be quite important.

\section{Two-valley motion}

This motion provides an extreme jump in pitch angle. The jump is so large that it could in some situations be the dominant mechanism for scattering particles into the loss cone. This motion involves $\phi_{0}=0$ and also having $\xi_{0}$ both 
finite and sufficiently large that $\xi_{0}^{2} / 2>\kappa^{\prime 2}$ so the coefficient of $\xi^{2}$ in Eq. (41) reverses sign. However, as will be shown below, there is an upper bound on $\xi_{0}$ that constrains the twovalleys to exist only for a certain finite range of $\xi_{0}$.

As sketched in Fig. 8(d) and shown quantitatively in Figs. 5(d) and 7(d), when the coefficient of $\xi^{2}$ is negative as happens if $\xi_{0}^{2} / 2>\kappa^{\prime 2}$, the pseudo-potential $\psi(\xi)$ has the form of two valleys separated by a hill. The pseudo-particle can only cross from one valley to the other if its pseudo-energy (right hand side of Eq. (42)) is sufficient to make it over the hill. In this case, the pseudo-particle can go back and forth between the two valleys. The left hand valley is a rough mirror image of the right hand valley, but is shallower because of the term linear in $\xi$ in Eq. (41). The upshot is that by making it over the hill and accessing the left hand valley, the particle can access a region of much larger negative $\xi$ than in the usual case. This corresponds to accessing a much larger pitch angle (i.e., closer to $180^{\circ}$ ). The spectacular nature of this effect can be seen by comparing the motion in Figs. 5 and 7 to the ordinary motion in Figs. 1 and 3. Figure 5 has $\phi_{0}=0, \kappa=1.6 \times 10^{-3}$, and initial $150^{\circ}$ pitch angle as for Figs. 1 and 3, but differs by having $\xi_{0}=0.031$ and now oscillates between $147^{\circ}$ and $168^{\circ}$; Fig. 7 is the same as Fig. 5 except that $\phi_{0}=180^{\circ}$, so the shape of $\psi(\xi)$ is the same, but the pseudo-particle starts with $d \xi / d t$ having the opposite sign. Figures 5 and 7 provide a remarkable $18^{\circ}$ transport of pitch angle towards the loss cone. It should be noted from Eq. (33) that the change in $\xi_{0}$ causes $n$ to be slightly different when $\xi_{0}$ is changed slightly.

The lower limit on $\xi_{0}$ for two valley motion is $\xi_{0}>$ $\sqrt{2} \kappa^{\prime}$. We now estimate the upper limit by first determining the height of the hill between the two valleys and then the condition for the total energy to exceed this height. The hill between the two valleys is near $\xi=0$, so the hill height is determined mainly by the competition between the quadratic and linear terms in $\psi(\xi)$ and so we approximate Eq. (41) as

$$
\psi(\xi) \simeq-\left(\frac{\xi_{0}^{2}}{2}-\kappa^{\prime 2}\right) \frac{\xi^{2}}{2}-\kappa^{\prime 2} \xi
$$

which has its maximum at

$$
\xi=-\kappa^{\prime 2} \frac{1}{\left(\frac{\xi_{0}^{2}}{2}-\kappa^{\prime 2}\right)}
$$

and the value of $\psi$ at this maximum (height of hill) is

$$
\psi_{\max } \simeq \frac{1}{2} \kappa^{\prime 4} \frac{1}{\left(\frac{\xi_{0}^{2}}{2}-\kappa^{\prime 2}\right)} .
$$

The question now is whether the total energy (kinetic plus potential) exceeds $\psi_{\max }$, so the particle can make it over the hill. The total energy is

$$
W=\frac{1}{2} s^{2} \kappa^{\prime 2}-\frac{1}{8} \xi_{0}^{4}+\kappa^{\prime 2} \frac{\xi_{0}^{2}}{2}-\kappa^{\prime 2} \xi_{0},
$$

and because we are seeking an upper limit on $\xi_{0}$, we assume that $\xi_{0}$ is much larger than $\kappa^{\prime}$ for this upper limit in which case

$$
W \simeq \frac{1}{2} s^{2} \kappa^{\prime 2}-\frac{1}{8} \xi_{0}^{4}
$$

and $\psi_{\max } \simeq 0$. The condition $W>\psi_{\max }$ then reduces to $W>0$ or

$$
\xi_{0}<4^{1 / 4}\left(s \kappa^{\prime}\right)^{1 / 2}
$$

Thus, two valley motion will occur when $\sin \phi_{0}=0$ and

$$
\sqrt{2} \kappa^{\prime}<\xi_{0}<4^{1 / 4}\left(s \kappa^{\prime}\right)^{1 / 2}
$$

Examination of the circumstances when two valley motion occurs shows that this energy requirement is indeed the case. When $\xi_{0}$ is increased, $\psi\left(\xi_{0}\right)$ becomes increasingly negative until the particle lacks sufficient energy to make it over the hill between the two valleys. The necking down of the phase-space curve in Figs. 5(c) and 7(c) shows the reduction in $\left|d \xi / d t^{\prime}\right|$ as the particle barely makes it over the hill separating the two valleys. The slight separation between the horizontal dotted line (total energy), and the hill peak in Figs. 5(d) and 7(d) shows how the particle has just enough energy to make it over the hill and access the valley on the left hand side.

\section{Left valley motion}

Examination of Eq. (41) shows that the coefficient of $\xi^{2}$ can also be made negative when $\phi_{0}=\pi / 2$ so again there is a valley to the left of $\xi=0$. If in this case, a particle starts with small negative $\xi_{0}$, it will be located near the right side of this left valley and will bounce in this left valley. It will not be able to access the right valley because its total pseudo-energy is near zero and so it cannot pass over the hill separating the two valleys. This motion is shown in Fig. 6 for $\phi_{0}=\pi / 2$ and $\xi_{0}=-0.031$.

\section{Extreme out of resonance motion}

If $\xi_{0}$ is extremely large, so the particle is very far from resonance, then Eq. (41) becomes

$$
\psi(\xi)=\frac{1}{8} \xi^{4}-\frac{\xi_{0}^{2}}{4} \xi^{2}
$$

which has minima at $\xi= \pm \xi_{0}$ and the value of $\psi$ at these minima is $\psi_{\min }=-\xi_{0}^{4} / 8$. This corresponds to a very deep well and yet the kinetic energy is only of the order of $s^{2} \kappa^{\prime 2}$ so the total energy $W$ is only slightly greater than the well minimum. The pseudo-particle then makes tiny bounces around the well minimum and so $\xi$ does not deviate significantly from $\xi_{0}$ when $\xi_{0}$ is extremely large.

\section{G. Bounce period}

If $\psi(\xi)$ has an approximate parabolic shape as for the $\xi_{0}=0$ cases with $\phi_{0}=0$ or $\pi$, the bounce period scaling can be determined to the extent that a Taylor expansion of $\psi(\xi)$ about its minimum is approximately valid. We define $\xi_{m}$ as the location of the well minimum, i.e., where $d \psi / d \xi=0$ 
and also define $\bar{\xi}=\xi-\xi_{m}$. Taylor expansion of $\psi(\xi)$ about its minimum gives

$$
\psi=\psi\left(\xi_{m}\right)+\frac{1}{2} \bar{\xi}^{2}\left(\frac{\partial^{2} \psi}{\partial \xi^{2}}\right)_{\xi_{m}},
$$

so Eq. (40) reduces to

$$
\frac{d^{2} \bar{\xi}}{d t^{\prime 2}}+\omega_{b}^{\prime 2} \bar{\xi}=0
$$

where

$$
\omega_{b}^{\prime}=\Omega^{\prime} \sqrt{\left(\frac{\partial^{2} \psi}{\partial \xi^{2}}\right)_{\xi_{m}}}
$$

is the bounce frequency in the wave frame.

The derivative of Eq. (41) is

$$
\frac{d \psi}{d \xi}=\frac{1}{2} \xi^{3}+\left(\kappa^{\prime 2}-\frac{\xi_{0}^{2}}{2}-s \kappa^{\prime} \sin \phi_{0}\right) \xi-\kappa^{\prime 2},
$$

so assuming $\kappa^{\prime}$ is small, $\sin \phi_{0}=0$, and $\xi_{0}=0$, setting $d \psi / d \xi=0$ gives the well minimum to be at

$$
\xi_{m} \simeq 2^{1 / 3} \kappa^{\prime 2 / 3} .
$$

The term linear in $\xi$ in Eq. (68) is negligible compared to the other two terms at $\xi=\xi_{m}$ and so has been neglected. The second derivative is

$$
\begin{aligned}
\left(\frac{d^{2} \psi}{d \xi^{2}}\right)_{\xi_{m}} & =\frac{3}{2} \xi_{m}^{2}+\left(\kappa^{\prime 2}-\frac{\xi_{0}^{2}}{2}-s \kappa^{\prime} \sin \phi_{0}\right) \simeq \frac{3}{2} \xi_{m}^{2} \\
& =\frac{3}{2}\left(2^{1 / 3} \kappa^{\prime 2 / 3}\right)^{2},
\end{aligned}
$$

since $\kappa^{\prime 2} \ll \kappa^{\prime 4 / 3}$. Using this second derivative in Eq. (67) gives the predicted bounce frequency to be

$$
\omega_{b}^{\prime}=\Omega^{\prime} \frac{\sqrt{3}}{2^{1 / 6}} \kappa^{\prime 2 / 3} .
$$

This predicted bounce frequency can be validated by comparison with the numerical calculations in Fig. 1. The number of cyclotron periods per bounce period for $\kappa^{\prime}=0.0016$ is predicted to be

$$
\frac{\Omega^{\prime}}{\omega_{b}^{\prime}} \simeq \frac{2^{1 / 6}}{\sqrt{3} \kappa^{\prime 2 / 3}}=\frac{2^{1 / 6}}{\sqrt{3}(0.0016)^{2 / 3}}=47 .
$$

Since $\Omega^{\prime}$ and $\omega_{b}^{\prime}$ are both frequencies, they have identical transformation rules for going from the wave frame to the lab frame, so it is predicted that

$$
\frac{\Omega}{\omega_{b}} \simeq 47,
$$

which is in excellent agreement with Fig. 1(e), which has about 47 cyclotron periods per bounce period. The temporal rate of change of pitch angle would be of the order of $\omega_{b}$ times the change in pitch angle. The $\kappa^{12 / 3}$ scaling of bounce frequency was verified numerically by varying $\kappa$ from $5 \times$ $10^{-4}$ to $5 \times 10^{-2}$ with $\xi_{0}=0$ and $\phi_{0}=0$ and then measuring the numerically calculated bounce frequency from plots similar to Fig. 1(e). Here, it is assumed that the relativistic correction is small so $\kappa^{\prime}$ is nearly the same as $\kappa$. These numerical solutions also showed that the increment $\Delta \theta$ in pitch angle relative to the initial pitch angle scales as $\kappa^{1 / 2}$. Thus, the rate of change of pitch angle due to bouncing in pseudo-potential wells scales as $\omega_{b} \Delta \theta \sim \kappa^{2 / 3} \times \kappa^{1 / 2}=\kappa^{7 / 6}$. This can be considered the approximate scaling for the single-pass rate at which coherent circularly polarized waves scatter the pitch angle of energetic electrons. In order to scatter the pitch angle, the wave packet duration must differ from an integral number of bounce periods, since otherwise the particle would simply return to its initial pitch angle.

When $\xi_{0} \neq 0$, so $\psi(\xi)$ has a two-valley shape, it is not appropriate to approximate $\psi(\xi)$ by Taylor expanding $\psi(\xi)$ about its minimum. Because of the complex shape of $\psi(\xi)$ in this situation, it is not possible to find a simple analytic expression for the bounce period. However, it is nevertheless possible to see intuitively what determines the bounce period. The bounce period in the wave frame can be defined as

$$
\tau^{\prime}=\oint \frac{d \xi}{d \xi / d t^{\prime}},
$$

so if there are extended portions of the phase-space orbit where $d \xi / d t^{\prime}$ is very small, the bounce period will become very long. This is evident in Figs. 5(c) and 7(c) where the phase-space orbit is necked down in the region near $\xi=0$. In this necked-down region, $\left|d \xi / d t^{\prime}\right|$ is small and so the pseudo-particle must spend a great deal of time covering a short distance in $\xi$ space, much like an automobile creeping along in a traffic jam. Figures 5(d) and 7(d) show that this small $\left|d \xi / d t^{\prime}\right|$ occurs when the total energy only slightly exceeds the potential energy (dotted line very close to the potential $\psi(\xi)$ ). Thus, the bounce period in the two valley situation will be some multiple of the bounce period for the single valley situation and will become infinite for the special situation where $W$ is infinitesimally larger than the peak of the hill between the two valleys.

\section{H. Pitch angle change as a function of mismatch}

Equation (1) shows that when $v_{z}$ changes, the particle will typically no longer be in resonance, i.e., $\xi$ will differ from being zero. Furthermore, Eq. (42) shows that $\xi$ can be considered as the displacement of a pseudo-particle bouncing in the potential well $\psi(\xi)$. The mismatch $\xi$ is directly related to $\beta_{z}$ via Eq. (30) while the normalized perpendicular velocity $\beta_{\perp}$ is a function of $\beta_{z}$ via the resonant diffusion curve, Eq. (17). These relations can be combined to express the pitch angle $\theta=\cos ^{-1}\left(\beta_{z} / \beta\right)$ as a function of $\xi$.

We start by using Eq. (17) to obtain $\beta_{\perp}$ and then $\beta$ in terms of $\beta_{z}$, so the pitch angle becomes a function of $\beta_{z}$, namely 


$$
\theta\left(\beta_{z}\right)=\cos ^{-1}\left(\frac{\varepsilon \beta_{z}}{\sqrt{\varepsilon^{2} \beta_{z}^{2}+\varepsilon^{2}-1+\frac{1}{n^{2} \Gamma}-\frac{1}{\Gamma}\left(\Gamma \beta_{z}-\frac{1}{n}\right)^{2}}}\right),
$$

where as before $\Gamma=n^{-2}+\varepsilon^{2}, n=c k / \omega$, and $\varepsilon=\left(1-\beta_{z 0} / n\right) /$ $\sqrt{1-\beta_{\perp 0}^{2}-\beta_{z 0}^{2}}$.

Next, Eq. (29) can be solved for $\beta_{z}^{\prime}$ to obtain

$$
\beta_{z}^{\prime}=\frac{\xi-1}{\sigma},
$$

where as before, $\sigma=\varepsilon \alpha n$ and $\alpha=\omega / \Omega$. Equations (13) and (14) can be expressed as

$$
\begin{gathered}
\varepsilon \beta_{z}^{\prime}=\gamma\left(\beta_{z}-\frac{1}{n}\right), \\
\varepsilon=\gamma\left(1-\frac{\beta_{z}}{n}\right),
\end{gathered}
$$

and upon dividing Eq. (77) by Eq. (78), we find

$$
\beta_{z}^{\prime}=\frac{\beta_{z}-\frac{1}{n}}{1-\frac{\beta_{z}}{n}},
$$

which is just the rule for relativistic velocity addition.

Combination of Eqs. (76) and (79) gives

$$
\beta_{z}(\xi)=n \frac{\xi-1+\varepsilon \alpha}{\xi-1+\varepsilon \alpha n^{2}},
$$

which for small $\xi$ is an offset linear function of $\xi$. The dependence of pitch angle on $\xi$ is then determined by inserting Eq. (80) in Eq. (75). Equations (75) and (80) show that the instantaneous value of pitch angle is a function of $\xi$ with $\varepsilon, \alpha$, and $n$ serving as parameters.

The total energy can be expressed using Eqs. (43), (46), and (41) as

$$
\begin{aligned}
W= & \frac{1}{2} s^{2} \kappa^{\prime 2} \cos ^{2} \phi_{0}+\frac{1}{8} \xi_{0}^{4} \\
& +\left(\kappa^{\prime 2}-\frac{\xi_{0}^{2}}{2}-s \kappa^{\prime} \sin \phi_{0}\right) \frac{\xi_{0}^{2}}{2}-\kappa^{\prime 2} \xi_{0} .
\end{aligned}
$$

The turning points of the pseudo-particle motion in the pseudo-potential $\psi(\xi)$ are the two real roots $\xi_{\min }, \xi_{\max }$ of the fourth-order polynomial

$$
\psi(\xi)-W=0 .
$$

Because $\psi(\xi)$ is a fourth order polynomial, there are in principle four roots to Eq. (82) and so a detailed prescription is needed to select the appropriate pair of roots that are the actual turning points. To do this, we define $\xi_{\min }$ as the leftmost real root and $\xi_{\max }$ the right-most real root for which $W>\psi(\xi)$ in the interval $\xi_{\min }<\xi_{0}<\xi_{\max }$. This choice of roots ensures that the pseudo-kinetic energy is positive definite in the interval, that the particle starts in this interval, and that the particle has sufficient energy to pass over any hill that might exist between two valleys. It will exclude inaccessible valleys.

The fourth order polynomial prescribed by Eq. (82) is

$$
\begin{aligned}
\frac{\xi^{4}}{8} & +\left(\kappa^{\prime 2}-\frac{\xi_{0}^{2}}{2}-s \kappa^{\prime} \sin \phi_{0}\right) \frac{\xi^{2}}{2}-\kappa^{\prime 2} \xi-\frac{s^{2} \kappa^{\prime 2}}{2} \cos ^{2} \phi_{0} \\
& -\frac{\xi_{0}^{4}}{8}-\left(\kappa^{\prime 2}-\frac{\xi_{0}^{2}}{2}-s \kappa^{\prime} \sin \phi_{0}\right) \frac{\xi_{0}^{2}}{2}+\kappa^{\prime 2} \xi_{0}=0 .
\end{aligned}
$$

Equation (83) must in general be solved numerically, but approximate solutions for certain regimes of interest were obtained in Sec. IIF. Rather than invoke approximate solutions, we have used a numerical root solver to find the four roots of Eq. (83) and then had a computer code identify which roots, if any, are complex. Because the coefficients in Eq. (83) are all real, complex roots occur in pairs that are complex conjugates of each other. If $\psi(\xi)$ has the profile sketched in Fig. 8(b), then Eq. (82) has only two real roots, but if $\psi(\xi)$ has the profile sketched in Fig. 8(d), then Eq. (82) will have four real roots if $W$ is less than the top of the hill between the two valleys and two real roots if $W$ is greater than the top of this hill. When the computer code detects that two of the four roots are complex, these two complex roots are discarded and the two real roots are defined to be $\xi_{\min }$ and $\xi_{\max }$ with $\xi_{\max }>\xi_{\min }$. These two roots are the $\xi$ turning points for situations as in Figs. 1-4. If four real roots exist, then the two intermediate roots bound the hill between two valleys and the particle is unable to move from the valley it started in, i.e., the other valley is inaccessible.

In order to investigate the parametric dependence of the motion, we vary $\xi_{0}$ while holding $\kappa^{\prime}$ and $s$ fixed to see how the turning points and $\psi(\xi)$ profiles change. The greatest increment in pitch angle towards the loss cone occurs at $\xi_{\min }$ since this gives the most negative value of $\beta_{z}$ in Eq. (80) and the most negative value of $\beta_{z}$ causes the pitch angle $\theta$ to move towards $180^{\circ}$. We also vary $\kappa^{\prime}$ while keeping $\xi_{0}$ constant.

Figure 9-11 each contain four pairs of plots that provide information on this parametric dependence. The upper plots in each pair show $\beta_{z}(\xi)$ plotted versus $\xi$ while the lower plot of each pair shows $\psi(\xi)$ plotted versus $\xi$. The lower plot also shows $W$ as calculated from Eq. $(81) . \psi(\xi)$ is shown as a solid curve only for the $\xi$ range where $W>\psi(\xi)$ and, in addition, the range includes $\xi_{0}$. Figure 9 shows plots where $\xi_{0}$ is incremented in relatively large steps while Fig. 10 shows the detailed behavior around the critical value of $\xi_{0}$ where two-valley profiles exist. Figure 10 shows the development of the two-valley structure as $\xi_{0}$ is increased and how the left-hand valley is inaccessible when $W$ is less than the height of the hill between the two valleys. The turning points $\xi_{\min }$ and $\xi_{\max }$ are identified by asterisks on the upper plots in Figs. 9 and 10. These upper plots show how the oscillation of $\xi$ maps into oscillation of $\beta_{z}$ which in turn maps into oscillation of pitch angle $\theta$. Figure 11 shows how pitch angle depends on $\kappa$ with $\xi_{0}$ fixed at zero.

Figure 12 is a contour plot where the increment in pitch angle relative to the initial assumed $150^{\circ}$ pitch angle is 

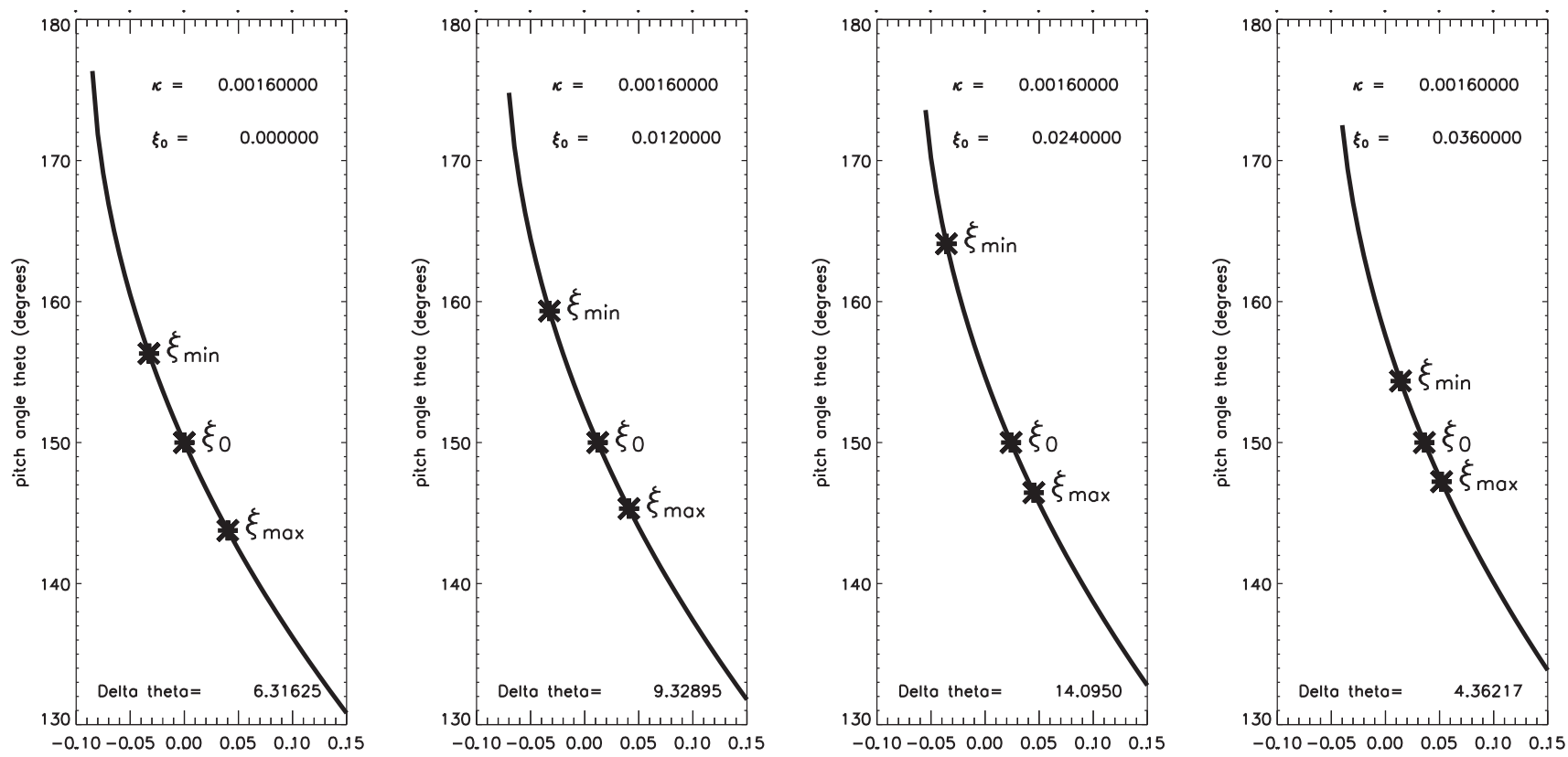

W
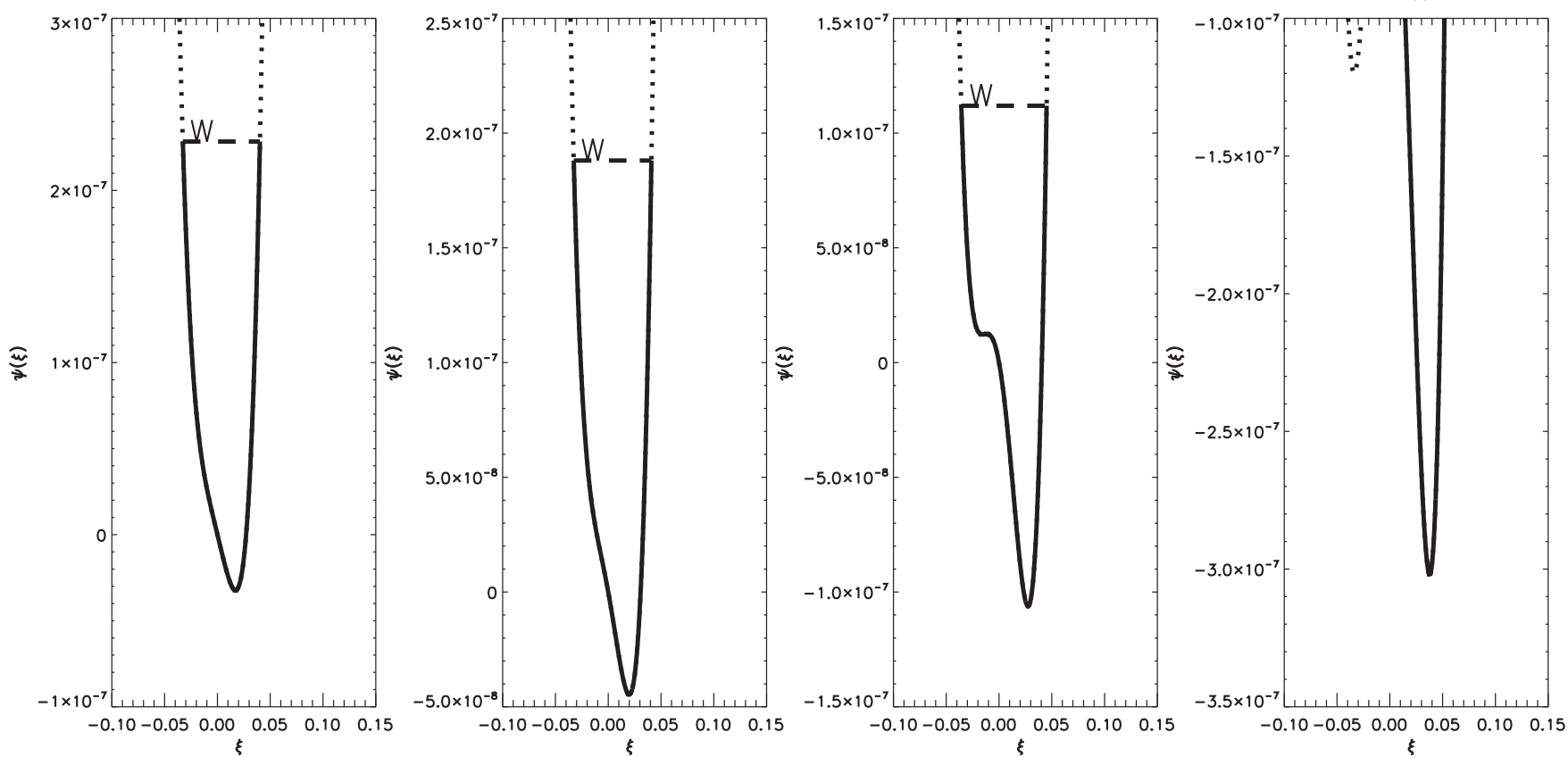

FIG. 9. Four pairs of upper/lower plots showing the dependence of pitch angle transport on variation of initial mismatch $\xi_{0}$ for fixed $\kappa$ and relatively large increments in $\xi_{0}$. The upper plot gives the mapping of $\xi$ to pitch angle, and the lower plot shows the pseudo-potential $\psi(\xi)$. The pseudo-potential $\psi(\xi)$ is shown as a solid line in the $\xi$ range when $\psi(\xi)<W$ and this range includes $\xi_{0}$ so it is accessible. Otherwise $\psi(\xi)$ is shown as a dotted line. $\xi_{0}$ and the turning points $\xi_{\min }$ and $\xi_{\max }$ in the lower plot (i.e., intersections of $\psi(\xi)$ with $W$ ) are shown as asterisks in the upper plot. The change in pitch angle from initial value to the value at $\xi_{\min }$ is presented at the bottom of the upper plots.

plotted as a function of $\xi_{0}$ and $\kappa$. For reference, the results of Fig. 9 are shown by the horizontal row of four diamonds, the results of Fig. 10 by the horizontal row of four x's, and the results of Fig. 11 as the vertical column of four squares. The abrupt termination of the two-valley motion occurring when the quasi-particle cannot make it over the hill appears as the slanting heavy line in the contour plots. The upper and lower limits prescribed by Eq. (63) are plotted as dashed lines. The right-most line corresponds to the upper limit and is nearly parallel to this ridge; it is slightly to the right of the ridge because the calculation for the upper limit was only done to lowest order in $\kappa^{\prime}$.
These pitch angle deflections are not extremely sensitive functions of $\phi_{0}$; running the numerical calculation with variation of $\phi_{0}$ by $\pm 10^{\circ}$ causes only slight change in the behavior.

\section{COMPARISON TO SPACECRAFT OBSERVATIONS}

Tsurutani et al. ${ }^{9}$ reported chorus observations where $B_{0}=125 \mathrm{nT}$ and $\tilde{B}=0.2 \mathrm{nT}$ giving normalized wave amplitude $\kappa=0.0016$. The wave period was $800 \mathrm{~Hz}$ and $\alpha=\omega / \omega_{c e}=0.25$. The parameters used in our numerical calculations were chosen to model this situation. Tsurutani 

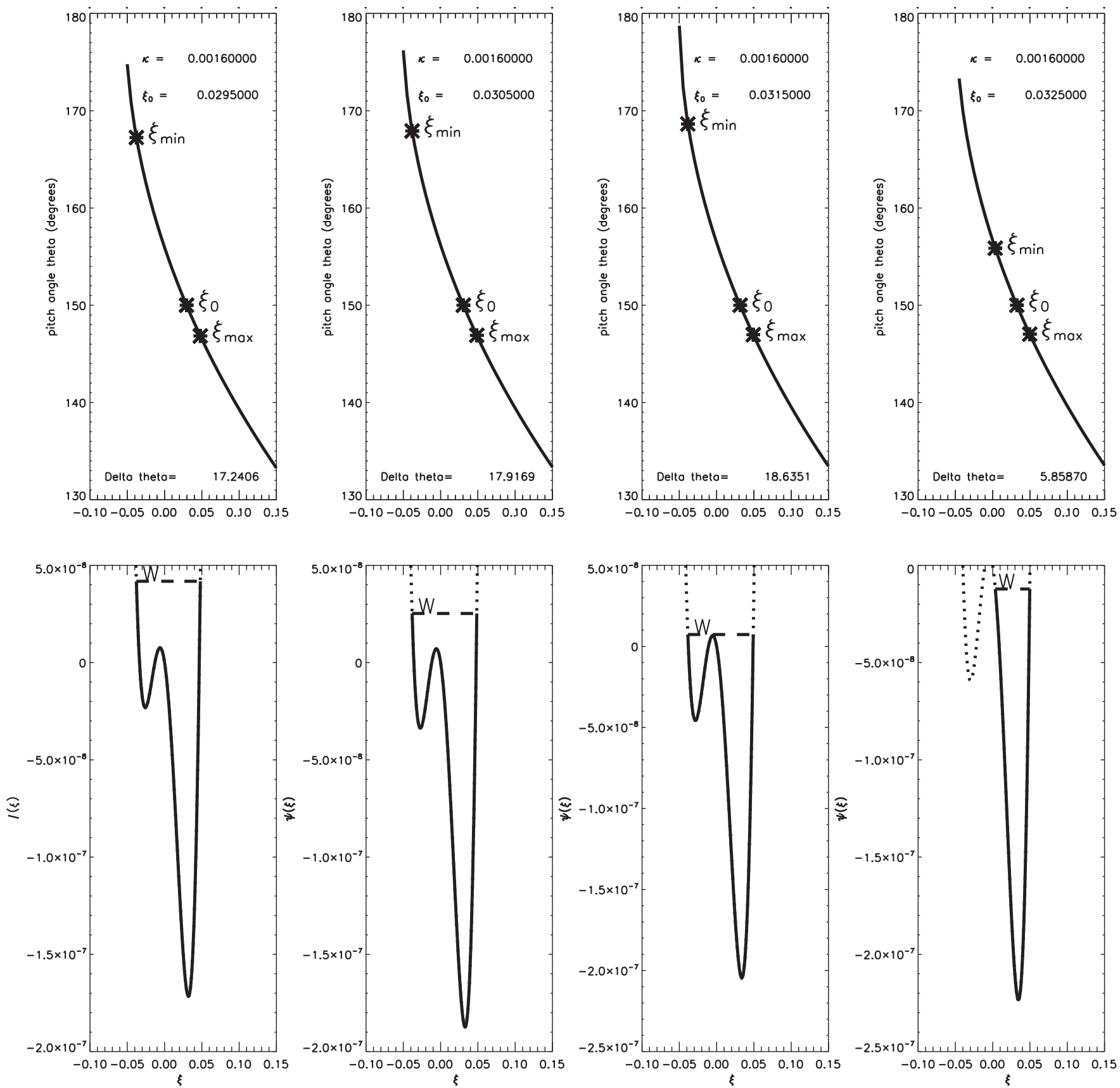

FIG. 10. Same as Fig. 8 except that here $\xi_{0}$ is varied in small increments in the vicinity of where the two-valley potential structure develops. The lower rightmost plot shows a two-valley situation where the left valley is inaccessible because the pseudo-particle does not have sufficient energy to pass over the hill separating the two valleys.

et al. ${ }^{9}$ reported that the chorus consisted of coherent subelements where each sub-element was a packet with coherent length of the order of $10 \mathrm{~ms}$. For example, Wave Packet 1 in Fig. 15 of Tsurutani et al. ${ }^{9}$ has a duration of $20 \mathrm{~ms}$ and is quite coherent from $2.584 \mathrm{~s}$ to $2.596 \mathrm{~s}$, i.e., is coherent for $12 \mathrm{~ms}$. During this coherence time, the $800 \mathrm{~Hz}$ wave has 10 periods, and these 10 periods will be moving at the phase velocity $c / n$. Because the wave has refractive index $n \approx 10$ and frequency $f=800 \mathrm{~Hz}$, the wavelength is $\lambda=c /(n f) \approx 38 \mathrm{~km}$, and the length of the packet is $L=c t / n=360 \mathrm{~km}$. The electron average parallel velocity as determined from Fig. $5(\mathrm{e})$ is $\beta_{z} \simeq-0.27$ or $v_{z} \approx$ $-8 \times 10^{7} \mathrm{~m} \mathrm{~s}^{-1}$ while the wave phase velocity is $c / n=$
$3 \times 10^{7} \mathrm{~m} \mathrm{~s}^{-1}$. Because the electron and the wave have oppositely directed velocities, the relative velocity is $c / n-v_{z}$. This relative velocity is the electron's approach velocity as would be seen by an observer in the wave frame. The time for the electron to transit the wave packet would then be

$$
\frac{L}{c / n-v_{z}}=\frac{t}{1-n \beta_{z}} \simeq \frac{0.012}{1+10 \times 0.27}=3 \mathrm{~ms} .
$$

This transit time corresponds to $0.003 \times f / \alpha \simeq 10$ cyclotron periods. Figure $3(\mathrm{~b})$ shows that an electron with $\xi_{0}=0$ and $\phi_{0}=180^{\circ}$ will have its pitch angle increase from $150^{\circ}$ to $156^{\circ}$ in approximately 10 cyclotron periods. Figure 6(b) 

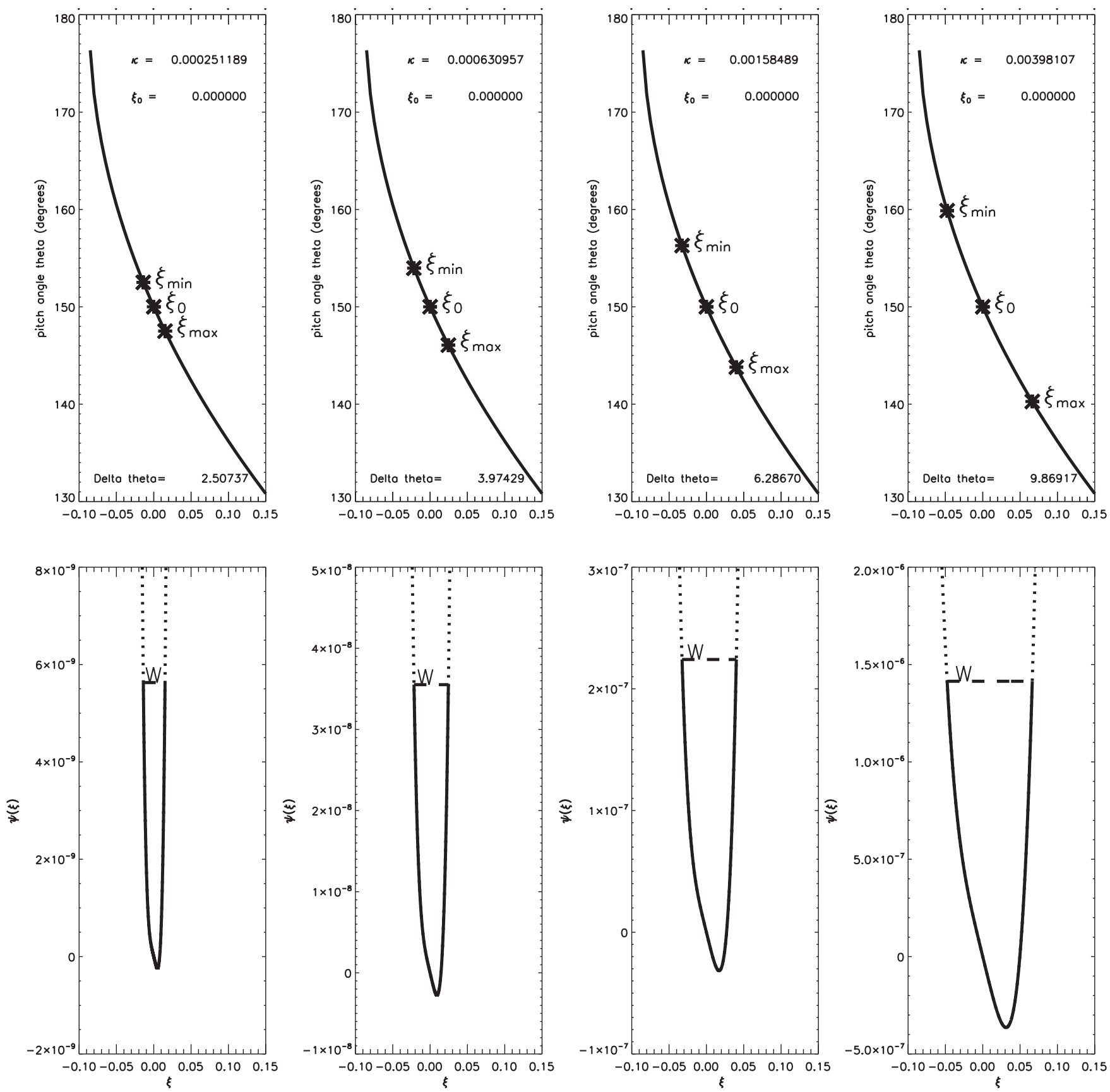

FIG. 11. Same as Figs. 8 and 9 except now $\xi_{0}=0$ and $\kappa$ is varied.

provides another example, this time with $\xi_{0}=-0.031$ and $\phi_{0}=\pi / 2$ where the electron increases its pitch angle by a similar amount in approximately 10 cyclotron periods. Fig. 7(b) provides a third example, this time with $\xi_{0}=0.031$ and $\phi_{0}=\pi$ where the electron increases its pitch angle by a similar amount in approximately 10 cyclotron periods. Thus, Figs. 3(b), 6(b), and 7(b) provide three distinct examples where an electron is deflected towards the loss cone by more than $5^{\circ}$ in a single pass through a wave having parameters corresponding to what has been measured by a spacecraft. If the electron were initially within $5^{\circ}$ of the loss cone, it would be scattered into the loss cone in a single pass through the wave. This would be a completely nondiffusive process.

\section{DISCUSSION}

We have examined the detailed motion of a particle moving in a coherent circularly polarized electromagnetic wave and a uniform background magnetic field. It is shown that this motion is highly sensitive to the initial phase angle $\phi_{0}$ relating the initial directions of the particle perpendicular velocity and the wave magnetic field. It is furthermore shown that the maximum possible single-pass change in pitch angle occurs when the particle is slightly off resonance, so an extra valley appears in the pseudo-potential; however, the packet duration has to be long enough for the particle to remain in the packet a half-bounce period. Figures 5 and 7 give examples where the particle jumps from a pitch angle 


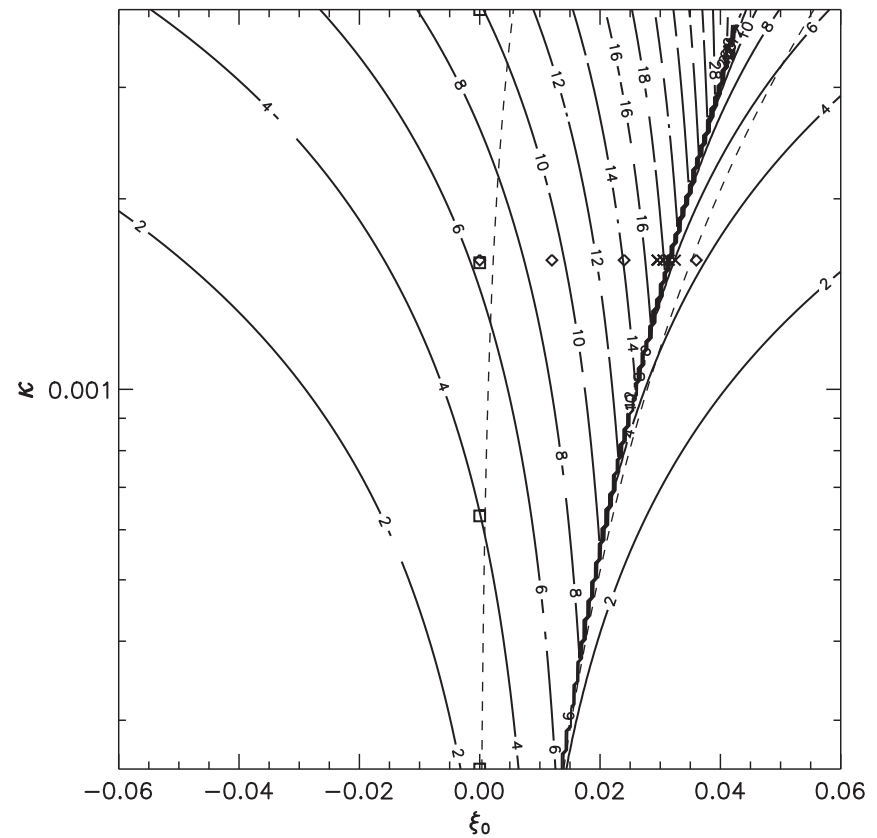

FIG. 12. Contours in $\xi_{0}, \kappa$ plane showing dependence on $\xi_{0}$ and on $\kappa$ of increment of pitch angle; this increment is relative to the $150^{\circ}$ initial pitch angle. The four cases shown in Fig. 9 (variation of $\xi_{0}$ at constant $\kappa$ ) are marked by diamonds, the four cases shown in Fig. 10 are marked by x's, and the four cases shown in Fig. 11 (variation of $\kappa$ at $\xi_{0}=0$ ) are shown by boxes. The upper and lower limits of $\xi_{0}$ for two-valley behavior as predicted by Eq. (63) are shown as dashed lines. The $\kappa$ scale (vertical axis) is logarithmic with base 10 .

of $150^{\circ}$ to a pitch angle of $168^{\circ}$ in 60 cyclotron periods (i.e., 15 wave periods since $\alpha=0.25$ is assumed) in a wave that has an amplitude that is just $0.16 \%$ of the background magnetic field. Depending on $\phi_{0}$ and $\xi_{0}$, the bounce phasing might be such that the particle pitch angle starts increasing immediately or starts decreasing. Figure 3, 6, and 7 show situations where the pitch angle immediately starts increasing, and so there is a pitch angle increase from $150^{\circ}$ to $155^{\circ}$ in just $3 \mathrm{~ms}$ for realistic parameters; these situations would be most important for short duration wave packets. One must be careful to realize that the time spent by the particle in the wave packet is not the same as the lifetime of the wave packet and it is the former time that matters from the point of view of the particle.

Equation (42) has the form of a classic oscillator but the shape of the potential function $\psi(\xi)$ is in general not parabolic. In general, the bounce frequency cannot be calculated simply by taking the second derivative of $\psi(\xi)$ as would be the case for a classic harmonic oscillator, but when $\psi(\xi)$ is approximately parabolic, this method gives a reasonable estimate. Just as a classic harmonic oscillator has an adiabatic invariant $J=\oint P d Q$, where $P$ and $Q$ are the conjugate canonical momentum and position; here, one would expect there to be an adiabatic invariant associated with the bounce motion in the potential well. This invariant would correspond to the area enclosed by the phase-space orbits in the (c) parts of Figs. 1-7. If there were some slowly varying parameter, one would expect the area enclosed by these phase-space orbits to remain invariant even though the orbit envelope shape would change. For example, if the wave frequency was to change slowly with respect to the bounce frequency in the $\psi$ valley, one would expect the (c) curves to deform in such a way so as to conserve enclosed area. This could result in greater variation in $\xi$ and hence more pitch angle transport. A simple measure of the spectral width $\Delta k$ in this situation of slowly changing wave frequency with conserved adiabatic invariant in $\left\{\xi,\left(1 / \Omega^{\prime}\right) d \xi / d t^{\prime}\right\}$ phase space would erroneously predict diffusion over the resonant diffusion curve when in fact there would be a regular, predictable motion.

Finally, it should be noted that when a particle moves leftward from its initial negative $\beta_{z}$ position in the (a) parts of Figs. 1-7 and so moves towards the loss cone, the particle loses energy as measured in the lab frame. This can be seen by noting that the resonant diffusion curves are essentially circles with centers displaced to the right of the origin in the (a) parts of Figs. 1-7 whereas surfaces of constant kinetic energy are circles centered at the origin of these curves. When a particle moves leftward from a negative $\beta_{z}$ starting point, it will intercept a constant $\beta=\sqrt{\beta_{z}^{2}+\beta_{\perp}^{2}}$ circle having a smaller radius than initially and so implying less kinetic energy. Thus, when a particle moves towards the loss cone, $\left|\beta_{z}\right|$ increases but there is a corresponding and larger decrease in $\beta_{\perp}$ with the net result that $\beta=\sqrt{\beta_{z}^{2}+\beta_{\perp}^{2}}$ decreases. If a wave-particle interaction decreases particle energy, then this energy must go into the wave and so the process of driving particles into the loss cone should result in strengthening the wave. This means that the overall system should be inherently unstable with respect to this behavior.

In conclusion, we have demonstrated some basic mechanisms whereby a particle can experience substantial pitch angle transport in a coherent wave and have given examples where there is a $5^{\circ}$ transport towards the loss cone in a single pass for observed wave parameters.

Consideration of the statistical distribution of these mechanisms is the next obvious step. We have provided some initial indication of the range of pitch angle scatterings that can occur by examining dependence on initial mismatch and on wave amplitude. Further examination of statistical behavior would require averaging over all possible $\phi_{0}$ 's, over all initial particle pitch angles, over a distribution of packet lengths/amplitudes/frequencies, and allowing for time-varying wave frequency.

\section{ACKNOWLEDGMENTS}

The author wishes to thank Dr. B. Tsurutani for raising the issue of whether coherent whistler wave chorus elements can cause significant pitch angle transport and for encouraging this analysis. This work was supported by NSF, DOE, and AFOSR.

${ }^{1}$ D. A. Gurnett and B. J. Obrien, J. Geophys. Res. 69, 65, doi:10.1029/ JZ069i001p00065 (1964).

${ }^{2}$ R. A. Helliwell, Whistlers and Related Ionospheric Phenomena (Stanford University Press, Stanford, 1965).

${ }^{3}$ C. T. Russell, R. E. Holzer, and E. J. Smith, J. Geophys. Res. 74, 755, doi:10.1029/JA074i003p00755 (1969).

${ }^{4}$ W. J. Burtis and R. A. Helliwell, J. Geophys. Res. 74, 3002, doi:10.1029/ JA074i011p03002 (1969). 
${ }^{5}$ B. T. Tsurutani and E. J. Smith, J. Geophys. Res. 79, 118, doi:10.1029/ JA079i001p00118 (1974).

${ }^{6}$ B. E. Goldstein and B. T. Tsurutani, J. Geophys. Res. 89, 2789, doi:10.1029/JA089iA05p02789 (1984).

${ }^{7}$ J. M. Albert, J. Geophys. Res. 106, 8477, doi:10.1029/2000JA000304 (2001).

${ }^{8}$ R. L. Stenzel, J. Geophys. Res. 104, 14379, doi:10.1029/1998JA900120 (1999).

${ }^{9}$ B. T. Tsurutani, O. P. Verkhoglyadova, G. S. Lakhina, and S. Yagitani, J. Geophy. Res. 114, A03207, doi:10.1029/2008JA013353 (2009).

${ }^{10}$ G. S. Lakhina, B. T. Tsurutani, O. P. Verkhoglyadova, and J. S. Pickett, J. Geophys. Res. 115, A00F15, doi:10.1029/2009JA014885 (2010).

${ }^{11}$ K. Min, J. Lee, and K. Keika, J. Geophy. Res. 115, A00I02, doi:10.1029/ 2010JA015474 (2010).

${ }^{12}$ Y. Zhang, W. W. Heidbrink, S. Zhou, H. Boehmer, R. McWilliams, T. A. Carter, S. Vincena, and M. K. Lilley, Phys. Plasmas 16, 055706 (2009).

${ }^{13}$ Y. Nariyuki, Phys. Plasmas 18, 052112 (2011).

${ }^{14}$ R. W. Boswell, Plasma Phys. Controlled Fusion 26, 1147 (1984).

${ }^{15}$ F. F. Chen and R. W. Boswell, IEEE Trans. Plasma Sci. 25, 1245 (1997).

${ }^{16}$ D. B. Melrose and G. A. Dulk, Astrophys. J. 259, 844 (1982).
${ }^{17}$ M. E. Mandt, R. E. Denton, and J. F. Drake, Geophys. Res. Lett. 21, 73, doi:10.1029/93GL03382 (1994).

${ }^{18}$ K. Fujimoto and R. D. Sydora, Geophys. Res. Lett. 35, L19112, doi:10.1029/2008GL035201 (2008).

${ }^{19}$ N. Singh, Phys. Rev. Lett. 107, 245003 (2011).

${ }^{20}$ S. Matsukiyo and T. Hada, Phys. Rev. E 67, 046406 (2003).

${ }^{21}$ R. A. Lopez, F. A. Asenjo, V. Munoz, and J. A. Valdivia, Phys. Plasmas 19, 082104 (2012).

${ }^{22}$ C. S. Roberts and S. J. Buchsbaum, Phys. Rev. 135, A381 (1964).

${ }^{23}$ H. R. Jory and A. W. Trivelpiece, J. Appl. Phys. 39, 3053 (1968).

${ }^{24}$ A. Holkundkar, G. Brodin, and M. Marklund, Phys. Rev. ST Accel. Beams 15, 091301 (2012).

${ }^{25}$ C. F. Kennel and H. E. Petschek, J. Geophys. Res. 71, 1, doi:10.1029/ JZ071i001p00001 (1966).

${ }^{26}$ R. C. Davidson, Methods in Nonlinear Plasma Theory (Academic Press, New York, 1972), pp. 54-56.

${ }^{27}$ A. Bourdier and S. Gond, Phys. Rev. E 62, 4189 (2000).

${ }^{28}$ B. L. Qian, IEEE Trans. Plasma Sci. 27, 1578 (1999).

${ }^{29}$ B. L. Qian, Phys. Plasmas 7, 537 (2000). 